\title{
RESEARCH
}

Open Access

\section{The role of Serpina3n in the reversal effect of ATRA on dexamethasone-inhibited osteogenic differentiation in mesenchymal stem cells}

Hai-Tao Jiang ${ }^{1,2+}$, Rui Deng ${ }^{1 \dagger}$, Yan Deng ${ }^{2,3}$, Mao Nie ${ }^{1}$, Yi-Xuan Deng ${ }^{2,3}$, Hong-Hong Luo ${ }^{2,3}$, Yuan-Yuan Yang ${ }^{2,3}$, $\mathrm{Na} \mathrm{Ni}{ }^{2,4}$, Cheng-Cheng $\operatorname{Ran}^{1,2}$ and Zhong-Liang Deng ${ }^{1 *}$ (D)

\begin{abstract}
Background: Glucocorticoid-induced osteoporosis (GIOP) is the most common secondary osteoporosis. Patients with GIOP are susceptible to fractures and the subsequent delayed bone union or nonunion. Thus, effective drugs and targets need to be explored. In this regard, the present study aims to reveal the possible mechanism of the anti-GIOP effect of all-trans retinoic acid (ATRA).

Methods: Bone morphogenetic protein 9 (BMP9)-transfected mesenchymal stem cells (MSCs) were used as an in vitro osteogenic model to deduce the relationship between ATRA and dexamethasone (DEX). The osteogenic markers runt-related transcription factor 2 (RUNX2), alkaline phosphatase (ALP), and osteopontin were detected using real-time quantitative polymerase chain reaction, Western blot, and immunofluorescent staining assay. ALP activities and matrix mineralization were evaluated using ALP staining and Alizarin Red S staining assay, respectively. The novel genes associated with ATRA and DEX were detected using RNA sequencing (RNA-seq). The binding of the protein-DNA complex was validated using chromatin immunoprecipitation (ChIP) assay. Rat GIOP models were constructed using intraperitoneal injection of dexamethasone at a dose of $1 \mathrm{mg} / \mathrm{kg}$, while ATRA intragastric administration was applied to prevent and treat GIOP. These effects were evaluated based on the serum detection of the osteogenic markers osteocalcin and tartrate-resistant acid phosphatase 5b, histological staining, and microcomputed tomography analysis.

(Continued on next page)
\end{abstract}

\footnotetext{
* Correspondence: dengz|@camu.edu.cn

${ }^{\dagger}$ Hai-Tao Jiang and Rui Deng contributed equally to this work.

'Department of Orthopaedics, The Second Affiliated Hospital of Chongqing

Medical University, 76 Linjiang Road, Yuzhong District, Chongqing 400010,

China

Full list of author information is available at the end of the article
}

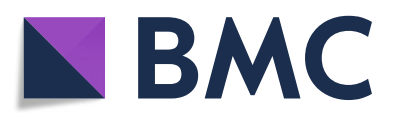

(- The Author(s). 2021 Open Access This article is licensed under a Creative Commons Attribution 4.0 International License, which permits use, sharing, adaptation, distribution and reproduction in any medium or format, as long as you give appropriate credit to the original author(s) and the source, provide a link to the Creative Commons licence, and indicate if changes were made. The images or other third party material in this article are included in the article's Creative Commons licence, unless indicated otherwise in a credit line to the material. If material is not included in the article's Creative Commons licence and your intended use is not permitted by statutory regulation or exceeds the permitted use, you will need to obtain permission directly from the copyright holder. To view a copy of this licence, visit http://creativecommons.org/licenses/by/4.0/. The Creative Commons Public Domain Dedication waiver (http://creativecommons.org/publicdomain/zero/1.0/) applies to the data made available in this article, unless otherwise stated in a credit line to the data. 
(Continued from previous page)

Results: ATRA enhanced BMP9-induced ALP, RUNX2 expressions, ALP activities, and matrix mineralization in mouse embryonic fibroblasts as well as C3H10T1/2 and C2C12 cells, while a high concentration of DEX attenuated these markers. When DEX was combined with ATRA, the latter reversed DEX-inhibited ALP activities and osteogenic markers. In vivo analysis showed that ATRA reversed DEX-inhibited bone volume, bone trabecular number, and thickness. During the reversal process of ATRA, the expression of retinoic acid receptor beta (RAR $\beta$ ) was elevated. RAR $\beta$ inhibitor Le135 partly blocked the reversal effect of ATRA. Meanwhile, RNA-seq demonstrated that serine protease inhibitor, clade A, member 3N (Serpina3n) was remarkably upregulated by DEX but downregulated when combined with ATRA. Overexpression of Serpina3n attenuated ATRA-promoted osteogenic differentiation, whereas knockdown of Serpina3n blocked DEX-inhibited osteogenic differentiation. Furthermore, ChIP assay revealed that RAR 3 can regulate the expression of Serpina3n.

Conclusion: ATRA can reverse DEX-inhibited osteogenic differentiation both in vitro and in vivo, which may be closely related to the downregulation of DEX-promoted Serpina3n. Hence, ATRA may be viewed as a novel therapeutic agent, and Serpina3n may act as a new target for GIOP.

Keywords: Glucocorticoid-induced osteoporosis (GIOP), Dexamethasone, ATRA, Serpina3n, BMP9, Osteogenic differentiation

\section{Background}

Glucocorticoid-induced osteoporosis (GIOP), the most common secondary cause of osteoporosis and the most common iatrogenic disease, is caused by the high dosage and the long-term use of glucocorticoids, such as dexamethasone and prednisone [1-4]. Glucocorticoids mainly inhibit osteogenesis by suppressing the differentiation of osteoblasts and the normal functioning of osteocytes, thus inducing their apoptosis $[5,6]$. On the contrary, the lifespan of osteoclasts can be prolonged and the ratio of receptor activator of nuclear factor- $\mathrm{kB}$ ligand to osteoprotegerin (RANKL/OPG) can be increased by glucocorticoids, leading to enhanced bone resorption [7-9]. Furthermore, glucocorticoids promote adipogenesis by enhancing the PPAR $\gamma$ and $\mathrm{C} / \mathrm{EBP} \alpha$ signaling pathways $[10,11]$. GIOP patients with fractures are susceptible to delayed bone union and nonunion [12]. Hence, new anti-GIOP agents and drugs need to be explored and their underlying mechanisms need to be elucidated to prevent and treat GIOP effectively.

Bone morphogenetic protein-9 (BMP9) belongs to transforming growth factor- $\beta$ (TGF $\beta$ ) superfamily. As one of the most potent BMPs involved in osteogenic differentiation, BMP9 exerts its function chiefly through the BMP/Smad signaling pathway to further activate Runt-related transcription factor-2 (RUNX2) and osteogenesis [13-15]. Thus, BMP9-induced osteogenic differentiation of mesenchymal stem cells (MSCs) can be regarded as in vitro osteogenic models. Several factors and signaling pathways, such as IGF1/2 [16, 17], COX-2 $[18,19]$, PTEN $[20]$, and $\mathrm{Wnt} / \beta$-catenin, are closely related to BMP9-induced osteogenic differentiation [21, 22]. In addition, BMP9 can also activate PI3K/AKT via the noncanonical pathway to regulate cancer cell proliferation, differentiation, invasion, and metastasis [23-25].
All-trans retinoic acid (ATRA) is the major biologically active form of vitamin A. ATRA and its isomers regulate cellular growth and differentiation by binding to their nuclear receptors, such as retinoic acid receptors (RARs) and retinoid $\mathrm{X}$ receptors (RXRs). Both receptors have three subtypes, namely, $\alpha, \beta$, and $\gamma[26,27]$. ATRA binds to RARs with high affinity but binds to RXRs only when its concentration is $>10^{-5} \mathrm{M}[28,29]$. Intracellular ATRA can be bound to cellular RA-binding proteins (CRABPs). ATRA bound to CRABPI is targeted for degradation by enzymes of the CYP26 family, whereas ATRA bound to CRABPII is further recruited into the nucleus and bound to RARs [30]. Our previous study indicated that ATRA can enhance BMP9-induced osteogenic differentiation and shift rosiglitazone-induced adipogenic differentiation to osteogenic differentiation [31, 32].

Serine protease inhibitor, clade A, member 3N (Serpina3n) is a novel inhibitory protein that was previously known as antichymotrypsin [33]. The role of Serpina3n has not yet been well-characterized. It has been reported that Serpina3n is highly expressed in brain, testis, lungs, thymus, and spleen; however, the expression of this protein is low in the bone marrow, skeletal muscle, and kidney [34]. Serpina3n is known to be involved in pathophysiological processes such as wound healing [35], pulmonary fibrosis [36], and muscle atrophy [37]. For osteogenic differentiation, Serpina3n has been reported to inhibit osteoblast phenotypes such as alkaline phosphatase (ALP), osteocalcin (OCN), and matrix mineralization [38], but little is known about the underlying mechanism. Therefore, further investigations are needed.

In this study, we combined the osteogenic inhibitory effect of DEX with the osteogenic promoting effect of ATRA. In a BMP9-induced osteogenic model, we found 
that the osteogenic inhibition is reversible by ATRA. We further discerned that DEX dramatically enhances the expression of Serpina3n, while in the reversal process, ATRA lowers Serpina3n expression. Furthermore, Serpina3n overexpression and knockdown confirmed that ATRA may reverse DEX-inhibited osteogenic differentiation via the attenuation of Serpina3n expression. Our findings may help in elucidating the mechanism of GIOP and in exploring novel anti-GIOP agents and drugs.

\section{Methods}

\section{Cell culture and chemicals}

HEK-293, C3H10T1/2, and C2C12 cells were purchased from American Type Culture Collection (ATCC, Manassas, VA, USA). The above cells were cultured in Dulbecco's modified Eagle's medium (DMEM), which contained $10 \%(\mathrm{v} / \mathrm{v})$ fetal bovine serum (FBS), penicillin $(100 \mathrm{U} / \mathrm{mL})$, and streptomycin $(100 \mu \mathrm{g} / \mathrm{mL})$. Cell culturing dishes, flasks, 6-well plates, and 24-well plates were incubated at $37^{\circ} \mathrm{C}$ with $5 \% \mathrm{CO}_{2}$. DMEM, FBS, and trypsin were purchased from Gibco (Chinese branch). BMP9 (sc-514211), ALP (sc-271431), RUNX2 (sc12488), glyceraldehyde-3-phosphate dehydrogenase (GAPDH, sc-32233) antibodies, retinoic acid receptor beta (RAR $\beta$ ) specific inhibitor Le135 (sc-204053), and ATRA (sc-200898) were bought from Santa Cruz Biotechnology (Chinese branch). Osteopontin (OPN, ab8448) and RAR $\beta$ (ab53161) antibodies were purchased from Abcam (Chinese branch). Serpina3n (AF4709) antibody was sourced from R\&D systems (Chinese branch). Dexamethasone (D4902) was obtained from SigmaAldrich (Chinese branch). Dylight 594 conjugated secondary antibody (A23410) was purchased from Abbkine (Chinese branch). Alkaline Phosphatase Assay Kit (C3206) and Alizarin Red S (ST1078) were bought from Beyotime (Shanghai, China).

\section{Construction of recombinant adenoviruses}

The recombinant adenoviruses used in this study were constructed according to Ad-Easy system [39, 40]. Briefly, the coding regions of the target genes green fluorescent protein (GFP), BMP9, red fluorescent protein (RFP), and Serpina3n were cloned into the shuttle vectors as well as the Serpina3n siRNA oligo fragments. The resultant plasmids were linearized by restriction endonuclease digestion. Subsequently, these plasmids were transformed into competent AdEasier cells containing the plasmid backbone of the adenovirus. Finally, the recombinant adenovirus plasmids were digested with PacI and transfected into HEK-293 cells for 14-20 days. The recombinant adenoviruses were designated as AdGFP, Ad-BMP9, Ad-RFP, Ad-Serpina3n, and AdsiSerpina3n [20, 41]. Among them, either Ad-GFP or
Ad-RFP was used as a vector control to track the virus lacking target gene expression.

\section{Isolation of mouse embryonic fibroblasts (MEFs)}

MEFs used in this study were isolated from post-coitus day 12.5 mice embryos, as described previously [42]. Briefly, each embryo was dissected and sheared using an 18 -gauge syringe in the presence of $1 \mathrm{~mL} 0.25 \%$ trypsin, followed by incubation for $15 \mathrm{~min}$ at $37^{\circ} \mathrm{C}$ with gentle shaking. Then, $10 \mathrm{~mL}$ DMEM containing $10 \%$ FBS was added to deactivate the trypsin. Subsequently, the dissected cells were plated in $10-\mathrm{cm}$ dishes and incubated at $37^{\circ} \mathrm{C}$ in the presence $5 \% \mathrm{CO}_{2}$ for $24 \mathrm{~h}$. Finally, the adherent cells were termed MEFs. All MEFs used in this study were within five passages.

\section{Osteogenic inductive medium (OIM) for osteogenic differentiation}

OIM consisted of DEX, sodium $\beta$-glycerophosphate, and $\mathrm{L}$-ascorbic acid. The three ingredients were dissolved in DMEM and filtered using a $0.2-\mu \mathrm{m}$ filter. The obtained solution was added to DMEM containing $10 \%$ FBS. The final concentration of DEX was $10^{-8} \mathrm{M}$, sodium $\beta$ glycerophosphate was $10 \mathrm{mM}$, and L-ascorbic acid was $250 \mu \mathrm{M}$. For osteogenic inductive cell culturing, the cells were seeded in 24-well plates and infected with adenoviruses after adhesion. After $36 \mathrm{~h}$, the medium was replaced by OIM, which was renewed once the color changed to light yellow (normally once a week) until the final Alizarin Red S staining (14 or 21 days later).

\section{RNA isolation, CDNA preparation, and real-time} quantitative polymerase chain reaction (RTqPCR) analysis At the corresponding timepoints, total RNA was extracted with TRIZOL reagent (Invitrogen, USA) and then purified and used for reverse transcription (RT) to generate cDNA. Later, the resultant cDNA was diluted 5-10 folds for RTqPCR assay. The expression of the housekeeping gene (GAPDH) were used to normalize the PCR results. ALP, RUNX2, RAR $\alpha, \operatorname{RAR} \beta, \operatorname{RAR} \gamma$, and Serpina3n were served as the target genes. The primer sequences used in this study are presented in Table 1.

\section{Protein harvesting and Western blot analysis}

The cells were previously seeded in 6-well plates and treated with different factors according to the experiment design. At the designed timepoint, the cells in each well of the 6-well plates were washed twice with cold phosphate buffered saline (PBS, $4{ }^{\circ} \mathrm{C}$ ) and lysed on ice with $300 \mu \mathrm{L}$ of lysis buffer containing protease and phosphatase inhibitors. Subsequently, the lysed cells were transferred into 1.5-mL Eppendorf tubes, followed by the addition of $75 \mu \mathrm{L}$ loading buffer and $15 \mu \mathrm{L} \beta$ mercaptoethanol in each tube. Then, the tubes were 
Table 1 Primers used in PCR assay

\begin{tabular}{|c|c|c|c|}
\hline Gene & Assay & Forward $\left(5^{\prime} \rightarrow 3^{\prime}\right)$ & Reverse $\left(5^{\prime} \rightarrow 3^{\prime}\right)$ \\
\hline GAPDH & PCR & ACCCAGAAGACTGTGGATGG & CACATTGGGGGTAGGAACAC \\
\hline ALP & PCR & AGACCAGGTCTGCTCAGGAT & ACCCCGCTATTCCAAACAGG \\
\hline RUNX2 & $P C R$ & GCCAATCCCTAAGTGTGGCT & AACAGAGAGCGAGGGGGTAT \\
\hline RARa & PCR & TCTCCCTGGACATTGACCTC & GTGTCTTGCTCAGGCGTGTA \\
\hline RAR $\beta$ & PCR & AATGCCACCTCTCATTCAGG & GAATGTCTGCAACAGCTGGA \\
\hline RARY & $P C R$ & AGGCAGCAGACTGACCATTT & TTCTGGTAGGTGTGCAGCAG \\
\hline Serpina3n & $P C R$ & GCTITTCTGCACTGTGGTGG & AGGCTGTAGTCGGTGGAGAT \\
\hline Serpina3n (primer 1) & ChIP & AAGGCAAGGGTGATACAGGC & TCCСTCATTGACCCCAAAGC \\
\hline Serpina3n (primer 2) & ChIP & CCCCCTTTCCACATCGACTC & TGTGCTCTGTGCTCAGACTG \\
\hline Serpina3n (primer 3) & ChIP & AGCCACTGAGCCTTCACTTC & ТTCСТTTСTCTCAACCCGCC \\
\hline
\end{tabular}

bathed in boiling water for $10 \mathrm{~min}$. These samples were separated via sodium dodecyl sulfate polyacrylamide gel electrophoresis, and the target gels were transferred to polyvinylidene difluoride (PVDF) membranes. The membranes were bathed in $5 \%$ bovine serum albumin (BSA) for $1 \mathrm{~h}$ at room temperature (RT) and then incubated in the corresponding antibody solutions at RT for $1.5 \mathrm{~h}$ or at $4{ }^{\circ} \mathrm{C}$ overnight. The membranes were washed five times using Tris-buffered saline Tween20 (TBST) and incubated in corresponding biotin-labeled immunoglobulin G (IgG, Beyotime, Shanghai, China) for $0.5 \mathrm{~h}$ at RT. Next, the membranes were also washed five times using TBST and incubated in horse radish peroxidase (HRP)-labeled streptavidin (Beyotime, Shanghai, China) for $0.5 \mathrm{~h}$ at RT. Finally, the membranes were washed five times with TBST, and the images of the target bands on the membranes were detected using SuperSignal West Pico Chemiluminescent Substrate (SWPCS) (Thermo Scientific, IL, USA). The antibodies were diluted in 5\% BSA according to the instructions (normally 1:1000), the biotin-labeled IgGs were diluted 1:3000 in TBST, and the HRP-labeled streptavidin was diluted 1:10,000 in TBST.

\section{Alkaline phosphatase staining assay}

The cells were previously seeded in 24-well plates and treated with different factors, followed by incubation for the designated number of days. Then, the cells in the 24-well plates were washed twice with PBS and stained using the Beyotime Alkaline Phosphatase Assay Kit. Briefly, the staining solution contained ALP buffer, BCIP, and NTB in the ratio of $10 \mathrm{~mL}: 33 \mu \mathrm{L}: 66 \mu \mathrm{L}$. The cells were added to $200 \mu \mathrm{L}$ of the staining solution and were stained for $15 \mathrm{~min}$ in the dark. The plates were dried and photographed using a fluorescence microscope (Nikon, Japan). These results were repeated in at least three independent experiments.

\section{Alizarin Red S staining assay}

The cells seeded in the 24-well plates were treated with different factors and incubated for 14 or 21 days. Subsequently, the cells were washed twice with PBS and fixed with $4 \%$ paraformaldehyde for $10 \mathrm{~min}$. Then, the cells were washed gently using $\mathrm{pH} 4.2 \mathrm{PBS}$ and were stained with $0.4 \%$ Alizarin Red S solution. Finally, the plates were scanned and the images were captured using a fluorescence microscope (Nikon, Japan). For quantification, the mineralized matrix was bathed in $10 \%$ acetic acid and the absorbance at $405 \mathrm{~nm}$ was measured using a microplate reader as previously described [18, 41]. These results were repeated in at least three independent experiments.

\section{Immunofluorescence staining assay}

The cells were firstly seeded in 48-well plates and treated with the corresponding factors, followed by incubation at $37^{\circ} \mathrm{C}$ for $24 \mathrm{~h}$. Then cells were washed twice with PBS and fixed with $4 \%$ paraformaldehyde for 10 min. Subsequently, they were bathed in $0.3 \%$ TritonX100 (Solarbio, Beijing, China) to increase the permeability of the cell membrane, followed by three washes with PBS. Then, they were blocked with goat serum (Beyotime, Shanghai, China) at RT for $1 \mathrm{~h}$ and later bathed in the target antibodies at $4{ }^{\circ} \mathrm{C}$ overnight. The antibodies were recycled on the next day, and the cells were washed thrice with PBS, followed by incubation entirely in the dark with Dylight 594 conjugated secondary antibody at $37^{\circ} \mathrm{C}$ for $1 \mathrm{~h}$. Then, the cells were washed thrice with PBS and stained with 4,6-diamino-2-phenyl indole (DAPI) for $5 \mathrm{~min}$. Finally, they were washed thrice with PBS and bathed in $80 \%$ glycerin for immunofluorescent image capture. The dilution rates were 1:200 for antibodies, 1:50 for the secondary antibody, and 1:10,000 for DAPI. These results were repeated in at least three independent experiments. 


\section{RNA sequencing assay}

The MEFs in the seven dishes were divided into seven groups: GFP + DMSO, GFP + DEX, GFP + ATRA, BMP9 + DMSO, BMP9 + DEX, BMP9 + ATRA, and BMP9 + DEX + ATRA. The cells were treated with the corresponding factors and cultured for $36 \mathrm{~h}$. For the first $12 \mathrm{~h}$, they were treated only with Ad-GFP or Ad-BMP9. DEX or ATRA was added $12 \mathrm{~h}$ after adenovirus infection. The concentration of DEX was $10^{-6} \mathrm{M}$ and that of ATRA was $1 \mu \mathrm{M}$. Then, the MEFs were washed twice with PBS and extracted on ice using TRIZOL agent (Invitrogen). The RNA-seq experiments were performed by Novogene Biotech Co. Ltd. (Beijing, China). Briefly, the sequencing libraries were generated using NEBNext UltraTM RNA Library Prep Kit for Illumina (NEB, USA) according to the manufacturer's instructions. The library quality was assessed using the Agilent Bioanalyzer 2100 system. Subsequently, the gene expression profiles of the seven groups were analyzed using the Illumina Hiseq platform, followed by data analysis of the differentially expressed genes.

\section{Chromatin immunoprecipitation assay}

ChIP was performed using the ChIP Assay Kit (Beyotime, Beijing, China) as previously described [43]. Briefly, the MEFs in the dishes were crosslinked with $1 \%$ formaldehyde (final concentration) for $10 \mathrm{~min}$. The reactions were terminated by the addition of glycine to a final concentration of $125 \mathrm{mM}$ for $5 \mathrm{~min}$. Subsequently, the cells were washed with cold PBS and lysed with SDS lysis buffer containing protease inhibitors. Then, the lysate was sonicated to generate 400-800-bp sequences, incubated with $5 \mathrm{M} \mathrm{NaCl}$ at $65^{\circ} \mathrm{C}$ for $4 \mathrm{~h}$, extracted with phenolchloroform, and precipitated with ethanol. One third of the lysate was stored at $-80^{\circ} \mathrm{C}$ to be used for subsequent PCR analysis, and the remaining two thirds were subjected to immunoprecipitation using RAR $\beta$ antibody or goat IgG. Finally, the precipitated protein-DNA complex was pulled down with Protein A + G Agarose/Salmon Sperm DNA, incubated with $5 \mathrm{M} \mathrm{NaCl}$, and extracted with phenol-chloroform for further PCR analysis. Goat IgG was employed as the negative control. Primers of Serpina3n used in the ChIP assay are listed in Table 1.

\section{Construction of GIOP rat model and ATRA treatment}

All animal experiments were approved by the Ethics Committee of Chongqing Medical University (CQMU). Forty 8-week-old male Sprague-Dawley (SD) rats weighing $200 \pm 10 \mathrm{~g}$ were obtained from the Animal Center of CQMU and were divided into eight groups as follows ( $n$ = 5): control group, GIOP group (DEX), DEX + prevention with low-dose ATRA group (DEX + LP), DEX + prevention with medium dose ATRA group (DEX +
MP), DEX + prevention with high-dose ATRA group $(\mathrm{DEX}+\mathrm{HP})$, DEX + treatment with low-dose ATRA group (DEX $+\mathrm{LT}), \mathrm{DEX}+$ treatment with medium-dose ATRA group (DEX + MT), and DEX + treatment with high-dose ATRA group (DEX + HT). After 1 week of acclimation, the rats were treated with the corresponding agents in the following manner: the rats in the DEXmediated groups (except for the control group) were intraperitoneally injected with DEX at a dose of $1 \mathrm{mg} / \mathrm{kg}$ (twice a week) for 8 weeks, and the rats in the control group were intraperitoneally injected with the same volume of normal saline for 8 weeks. ATRA was ground with sodium carboxymethyl cellulose and dissolved in water (in the high dose group, ATRA might not have been completely dissolved). In the ATRA prevention group, the rats were intragastrically administrated with ATRA for 8 weeks: $0.2 \mathrm{mg} / \mathrm{kg} /$ day for the LP group, 1.0 $\mathrm{mg} / \mathrm{kg} /$ day for the MP group, and $5.0 \mathrm{mg} / \mathrm{kg} / \mathrm{day}$ for the HP group. In the ATRA treatment group, the rats were intragastrically administrated with ATRA for 4 weeks after DEX injection: $0.2 \mathrm{mg} / \mathrm{kg} /$ day for the LT group, 1.0 $\mathrm{mg} / \mathrm{kg} /$ day for the MT group, and $5.0 \mathrm{mg} / \mathrm{kg} /$ day for the HT group. After 8 weeks, the rats were sacrificed and the blood and lumbar vertebrae of each group were collected for further serological and histological evaluations and micro-computed tomography $(\mu \mathrm{CT})$ analysis.

\section{Estimation of the serum concentration of bone metabolism-related markers}

The collected tubes of blood were coagulated for $30 \mathrm{~min}$ and centrifuged at $4{ }^{\circ} \mathrm{C}$ for $10 \mathrm{~min}$, and the supernatants (sera) were retrieved. Serum ALP $(405 \mathrm{~nm})$, calcium $(660 \mathrm{~nm})$, and phosphorus $(340 \mathrm{~nm})$ concentrations were estimated by using the corresponding kits. The absorbance values were measured using a microplate reader, and the optical density (OD) values were converted to concentration. For serum $\mathrm{OCN}$ and tartrate-resistant acid phosphatase 5b (TRACP-5b), enzyme-linked immunosorbent assay (ELISA) was employed for concentration detection via the corresponding kit. Briefly, the obtained OD values were calibrated using the standard sample and were converted to the concentrations according to the formula derived from the standard sample.

\section{Histological evaluation and staining}

The obtained lumbar vertebrae of the rats were fixed with $4 \%$ paraformaldehyde for 1 week, decalcified with EDTA solution ( $\mathrm{pH}$ 7.2) for 4 weeks, dehydrated with ethanol, and embedded with paraffin. Subsequently, the paraffin-coated masses were sliced and subjected to hematoxylin-eosin (H\&E) staining and Masson's trichrome staining after deparaffinization and rehydration. 


\section{Micro-computed tomographic analysis and 3D reconstruction}

All harvested lumbar vertebral samples were scanned with $\mu \mathrm{CT}$ (VivaCT 40, SCANCO Medical AG, Switzerland). The transverse plane, 3D reconstructions, and ROI analysis, such as the ratio of bone volume to total volume (BV/TV), trabecular number (Tb.N), trabecular thickness (Tb.Th), and trabecular separation (Tb.Sp), were performed using the software linked to the scanner ( $\mu$ CT 516.1).

\section{NIH ImageJ for quantification of protein bands and ALP activities}

The software NIH ImageJ (http://rsbweb.nih.gov/ij/ download.html) was used to quantify the protein bands and the ALP staining images. For the protein bands in Fig. 1, the ratios of the target protein to GAPDH normalized to the control (GFP + DMSO) are shown beneath the target bands. For the protein bands in other figures, the triplicated results were quantified, normalized to their control, and subjected to further statistical analysis. For ALP quantification, the images were blackwhite colored and color inverted, and each well was measured for further statistical analysis.

\section{Statistical analysis}

For statistical analysis, GraphPad Prism 6 software was used to analyze the standard deviations and the differences between two groups via two-tailed Student's $t$ test. $p$ values $<0.05$ were considered significant, and $p$ values $<0.01$ were considered remarkably significant. For all quantitative analysis, the experiments were carried out in triplicates, and the results were repeated in at least three independent experiments.

\section{Results}

\section{Effects of ATRA on BMP9-induced and OIM-induced} osteogenic differentiation of MEFs

Although the effect of ATRA on osteoblasts and osteocytes remains controversial, several research findings have demonstrated its positive effect on the osteogenic differentiation of MSCs. Therefore, we firstly checked the effect of ATRA on the osteogenic differentiation of MEFs. ALP staining assay showed that ATRA can induce ALP activities in a concentration-dependent manner (Fig. 1a, b). Next, we evaluated the effect of ATRA on matrix mineralization. Alizarin Res $\mathrm{S}$ staining and quantification established that ATRA alone cannot induce matrix mineralization (Fig. S1A-B and Fig. 1c, d). Subsequently, we utilized adenovirus-mediated BMP9 and validated its function (Fig. 1e, f). BMP9 was capable of solely inducing ALP activities; in addition, ATRA enhanced the ALP activities induced by BMP9 in a statistically significant manner (Fig. 1g, h). This effect was confirmed in C3H10T1/2 and C2C12 cells (Fig. S1C-H). RTqPCR and Western blot results of the osteogenic markers ALP and RUNX2 were consistent with those of ALP staining (Fig. 1i, j). Furthermore, Alizarin Red S staining confirmed that ATRA can enhance BMP9induced osteogenic differentiation (Fig. 1k and Fig. S1IJ). Finally, we replaced IOM with BMP9, and ATRA was found to promote OIM-induced ALP activities as well (Fig. 1l, m). These results suggest that ATRA can induce ALP activities but not matrix mineralization on their own. Meanwhile, ATRA can enhance both BMP9induced and OIM-induced osteogenic differentiation.

\section{Effects of DEX on BMP9-induced and OIM-induced osteogenic differentiation of MEFs}

Based on the knowledge that a high concentration of DEX inhibits osteogenesis, we used different concentrations of DEX to explore its effects on BMP9-induced and OIM-induced osteogenic differentiation. At a concentration of $10^{-9} \mathrm{M}$ and $10^{-8} \mathrm{M}, \mathrm{DEX}$ showed no obvious inhibition of BMP9-induced ALP activities; however, at $10^{-6} \mathrm{M}$, it resulted in the significant inhibition (Fig. 2a-c and Fig. S2A-F) of different MSCs. Western blot analysis and the results of quantification revealed that high concentrations of DEX $\left(10^{-7} \mathrm{M}\right.$ and $\left.10^{-6} \mathrm{M}\right)$ can attenuate the protein expressions of the early osteogenic markers RUNX2 and ALP and the late osteogenic marker OPN induced by BMP9 (Fig. 2d, e). Alizarin Res S staining and quantification also demonstrated that high concentrations of DEX inhibited BMP9-induced matrix mineralization (Fig. 2f-h). Given that DEX is an ingredient of OIM, we altered its concentration in OIM instead of adding different concentrations of it to OIM-induced MEFs. ALP and Alizarin Red $S$ staining and the quantifications demonstrated that high concentrations of DEX reduced ALP activities and matrix mineralization (Fig. 2i-1). These data imply that high concentrations of DEX can inhibit BMP9-induced and OIM-induced osteogenic differentiation.

\section{Effects of ATRA on DEX-inhibited osteogenic differentiation}

Next, we aimed to study the combined effect of ATRA and DEX on BMP9-induced osteogenic differentiation. To accurately evaluate the effect of ATRA, $10^{-6} \mathrm{M}$, which is the most effective concentration of DEX, was chosen for the following experiments. ALP staining indicated that ATRA reversed DEX-inhibited ALP activities and that their combined effects were stronger than those of the BMP9 alone group (Fig. 3a, b). The osteogenic markers ALP, RUNX2, and OPN (Fig. 3c-f) and matrix mineralization (Fig. 3g, h) inhibited by DEX were also reversed by ATRA. Subsequently, we utilized immunofluorescence staining, and the results revealed that the 


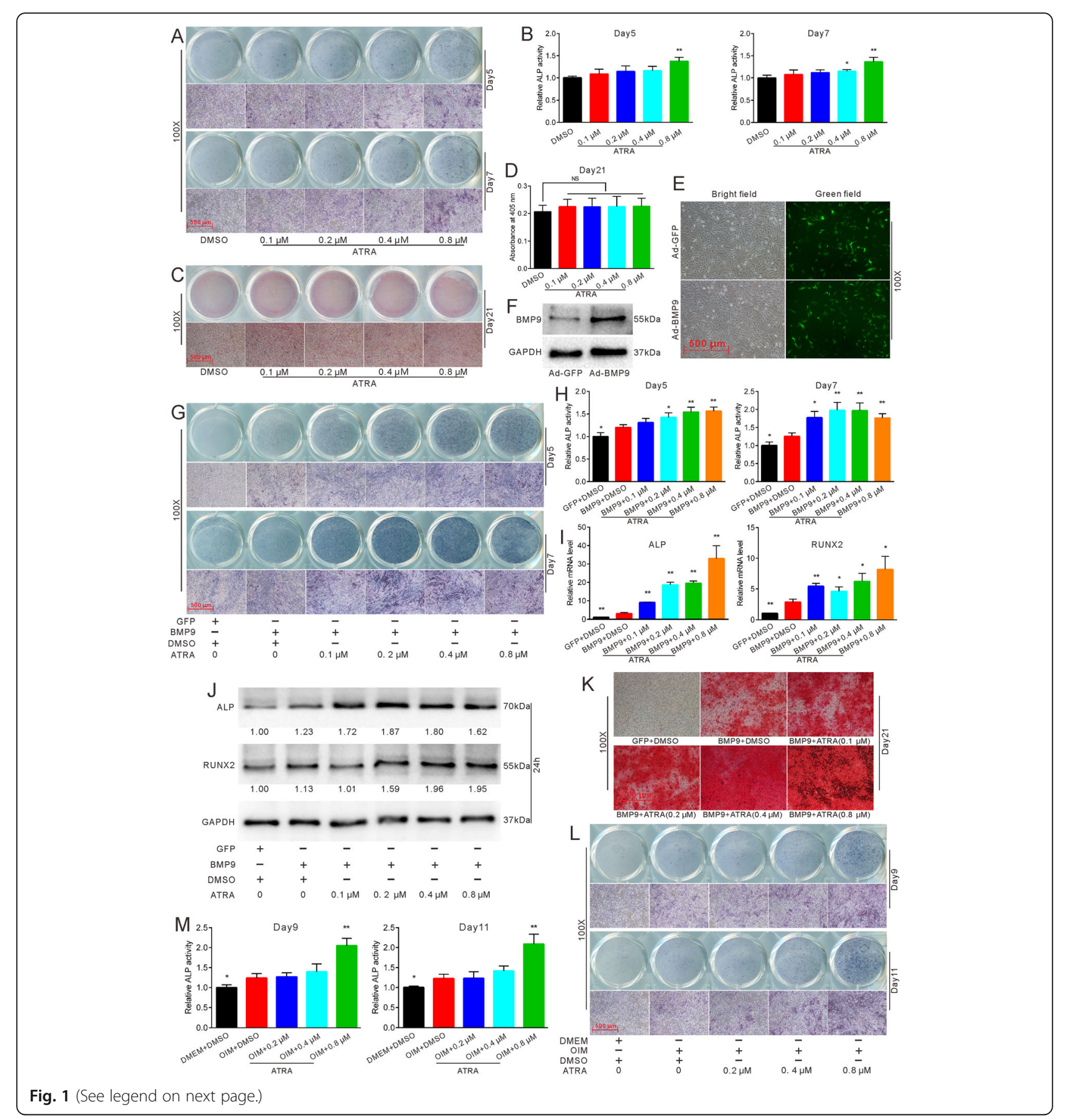


(See figure on previous page.)

Fig. 1 Effects of ATRA on osteogenic differentiation in MEFs. a ALP staining showed the effect of different concentrations of ATRA on ALP activities in MEFs after 5 and 7 days. $\mathbf{b}$ Quantification of ALP staining demonstrated the effect of different concentrations of ATRA on ALP activities in MEFs after 5 and 7 days, ${ }^{*} p<0.05$ vs DMSO, ${ }^{* *} p<0.01$ vs DMSO. c Alizarin Red S staining showed the effect of different concentrations of ATRA on matrix mineralization in MEFs after 21 days. d Quantification of matrix mineralization in MEFs after 21 days, ${ }^{*} p<0.05$ vs DMSO, ${ }^{* *} p<0.01$ vs DMSO. e MEFs infected with adenovirus-mediated Ad-GFP and Ad-BMP9 after $24 \mathrm{~h}$. $\mathbf{f}$ Western blot demonstrated the protein expression of BMP9 in MEFs $24 \mathrm{~h}$ after Ad-GFP and Ad-BMP9 infection. $\mathbf{g}$ ALP staining showed the effect of different concentrations of ATRA on BMP9-induced ALP activities in MEFs after 5 and 7 days. $\mathbf{h}$ Quantification of ALP staining showed the effect of different concentrations of ATRA on BMP9-induced ALP activities in MEFs after 5 and 7 days, ${ }^{*} p<0.05$ vs BMP9 + DMSO, ${ }^{* *} p<0.01$ vs BMP9 + DMSO. i RTqPCR showed the effect of different concentrations of ATRA on BMP9-induced osteogenic markers after $24 \mathrm{~h},{ }^{*} p<0.05$ vs BMP9 + DMSO, ${ }^{* *} p<0.01$ vs BMP9 + DMSO. $\mathbf{j}$ Western blot results showed the effect of different concentrations of ATRA on BMP9-induced osteogenic markers after $24 \mathrm{~h}$ (numbers between the bands were the ratios of target bands to GAPDH using ImageJ). $\mathbf{k}$ Microscopic view of Alizarin Red S staining showed the effect of different concentrations of ATRA on BMP9-induced matrix mineralization in MEFs after 21 days. I ALP staining showed the effect of different concentrations of ATRA on OIM-induced ALP activities in MEFs after 9 and 11 days. $\mathbf{m}$ Quantification of ALP staining demonstrated the effect of different concentrations of ATRA on OIM-induced ALP activities in MEFs after 9 and 11 days, ${ }^{*} p<0.05$ vs OIM + DMSO, ${ }^{* *} p<0.01$ vs OIM + DMSO. GFP, green fluorescent protein; DMSO, dimethyl sulfoxide; DMEM, Dulbecco's modified Eagle's medium; OIM, osteogenic

inductive medium

fluorescence of RUNX2 was stronger in the DEX and ATRA group when compared with the DEX alone group (Fig. 3i). We then employed C3H10T1/2 cells for further experiments. ALP staining illustrated that ATRA can reverse the inhibition of ALP activities caused by different concentrations $\left(10^{-9}-10^{-6} \mathrm{M}\right)$ of DEX (Fig. S3A-E). These results allude that ATRA can reverse DEXinhibited osteogenic differentiation in different MSCs.

\section{Effects of ATRA on bone metabolism in the GIOP rat model}

To further investigate the effects of ATRA on DEXinhibited osteogenesis, we constructed a GIOP rat model for prevention and treatment using ATRA. Estimation of the serum concentrations of ALP, calcium, and phosphorus revealed that DEX lowered the serum concentration of ALP. Both prevention and treatment using ATRA reversed DEX-lowered ALP concentration (Fig. 4a). However, both DEX and ATRA showed no effect on the serum concentrations of calcium and phosphorus (Fig. 4b, c). ELISA results demonstrated that the serum concentration of the osteoblastic marker OCN downregulated by DEX was reversed by ATRA in the prevention and treatment groups (Fig. 4d). In contrast, the serum osteoclastic marker TRACP-5b was enhanced by DEX but attenuated when combined with ATRA (Fig. 4e). H\&E and Masson's trichrome staining showed that the number and thickness of the trabecular bone were dramatically decreased by DEX and were partly reversed by the ATRA combination (Fig. 4f), irrespective of whether the vertebrae were from the prevention group or the treatment group. Furthermore, $\mu \mathrm{CT}$ scan, 3D reconstruction, and ROI analysis revealed that the bone trabecular changes in each group were in accordance with the histological evaluations (Fig. 4g, h). These data suggest that ATRA can reverse GIOP in the rat model, both in the prevention and treatment groups.

\section{Effects of RAR $\beta$ on the reversal process of ATRA with regard to DEX-inhibited osteogenic differentiation of MEFs}

Based on the knowledge that low concentrations of ATRA only functionally bind to RARs, we assessed the three types of RARs (RAR $\alpha, \operatorname{RAR} \beta$, and RAR $\gamma)$. We found that RAR $\beta$ was obviously upregulated by ATRA (Fig. 5a). Next, we checked the expression of RAR $\beta$ using different concentrations of ATRA. Western blot results and quantifications showed that RAR $\beta$ was upregulated by ATRA in a concentration-dependent manner (Fig. 5b, c). We exploited Le135, a specific RAR $\beta$ inhibitor, and found that ATRA-induced ALP activities can be attenuated by it (Fig. $5 \mathrm{~d}$, e). In addition, Le135 also attenuated ATRA-reversed protein expressions of the osteogenic markers RUNX2 and ALP (Fig. 5f, g), ALP activities (Fig. 5h, i), and matrix mineralization (Fig. $5 j$ ). These results suggest that ATRA may reverse DEXinhibited osteogenic differentiation by binding to RAR $\beta$ and upregulating its expression.

\section{Effects of DEX and ATRA on the expression of Serpina3n in MEFs}

To further explore the reversal mechanism of ATRA, we performed RNA-seq and found the novel gene Serpina3n, which has not been well-characterized so far. Western blot results and quantifications demonstrated that DEX can upregulate the protein expression of Serpina3 $\mathrm{n}$ in a concentration-dependent manner (Fig. 6a, b). On the contrary, ATRA lowered the expression of Serpina3n (Fig. 6c, d). Next, we found that the expression of Serpina3n was also promoted by DEX and inhibited by ATRA through the mediation of BMP9 (Fig. 6e, f). Moreover, the results of immunofluorescence staining were consistent with those of Western blot analysis (Fig. $6 \mathrm{~g})$. These data imply that Serpina3n can be regulated by DEX and ATRA. Meanwhile, Serpina3n may be 

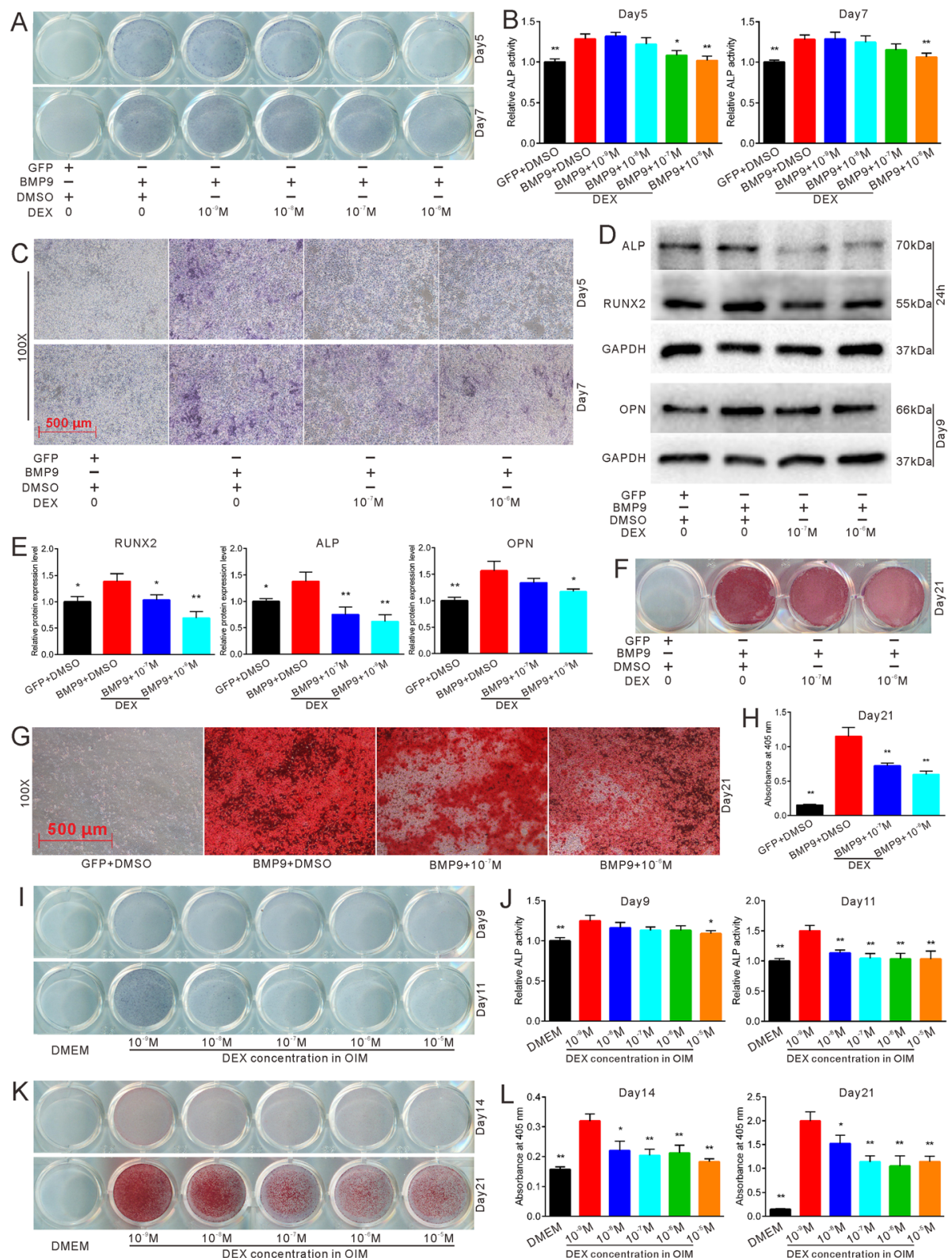

Fig. 2 Effects of DEX on osteogenic differentiation in MEFs. a ALP staining demonstrated the effect of different concentrations of DEX on BMP9induced ALP activities in MEFs after 5 and 7 days. $\mathbf{b}$ Quantification of ALP staining demonstrated the effect of different concentrations of DEX on BMP9-induced ALP activities in MEFs after 5 and 7 days, ${ }^{*} p<0.05$ vs BMP9 + DMSO, ${ }^{* *} p<0.01$ vs BMP9 + DMSO. c Microscopic view of ALP staining showed the effect of different concentrations of DEX on BMP9-induced ALP activities in MEFs after 5 and 7 days. $\mathbf{d}$ Western blot results showed the effect of different concentrations of DEX on BMP9-induced osteogenic markers. e Quantification of western blot demonstrated the effect of different concentrations of DEX on BMP9-induced osteogenic marker ALP, RUNX2, and OPN, ${ }^{*} p<0.05$ vs BMP9 + DMSO, ** $p<0.01$ vs BMP9 + DMSO. f Alizarin Red S staining showed the effect of different concentrations of DEX on matrix mineralization in MEFs after 21 days. $\mathbf{g}$ Microscopic view of Alizarin Red S staining demonstrated the effect of different concentrations of DEX on BMP9-induced matrix mineralization. $\mathbf{h}$ Quantification of matrix mineralization in MEFs, ${ }^{*} p<0.05$ vs BMP9 + DMSO, ${ }^{* *} p<0.01$ vs BMP9 + DMSO. i ALP staining demonstrated the effect of different concentrations of OIM on ALP activities in MEFs after 9 and 11 days. $\mathbf{j}$ Quantification of ALP staining showed the effect of different concentrations of OIM on ALP activities, ${ }^{*} p<0.05$ vs OIM with $10^{-9} \mathrm{M}$ DEX, ${ }^{* *} p<0.01$ vs OIM with $10^{-9} \mathrm{M}$ DEX. $\mathbf{k}$ Alizarin Red $\mathrm{S}$ staining demonstrated the effect of different concentrations of OIM on matrix mineralization in MEFs after 14 and 21 days. I Quantification of Alizarin Red $S$ staining showed the effect of different concentrations of OIM on matrix mineralization, ${ }^{*} p<0.05$ vs OIM with $10^{-9} \mathrm{M}$ DEX, ${ }^{* *} p<0.01$ vs OIM with $10^{-9} \mathrm{M}$ DEX. GFP, green fluorescent protein; DMSO, dimethyl sulfoxide; DEX, dexamethasone; OPN, osteopontin, DMEM, Dulbecco's modified Eagle's medium; OIM, osteogenic inductive medium 

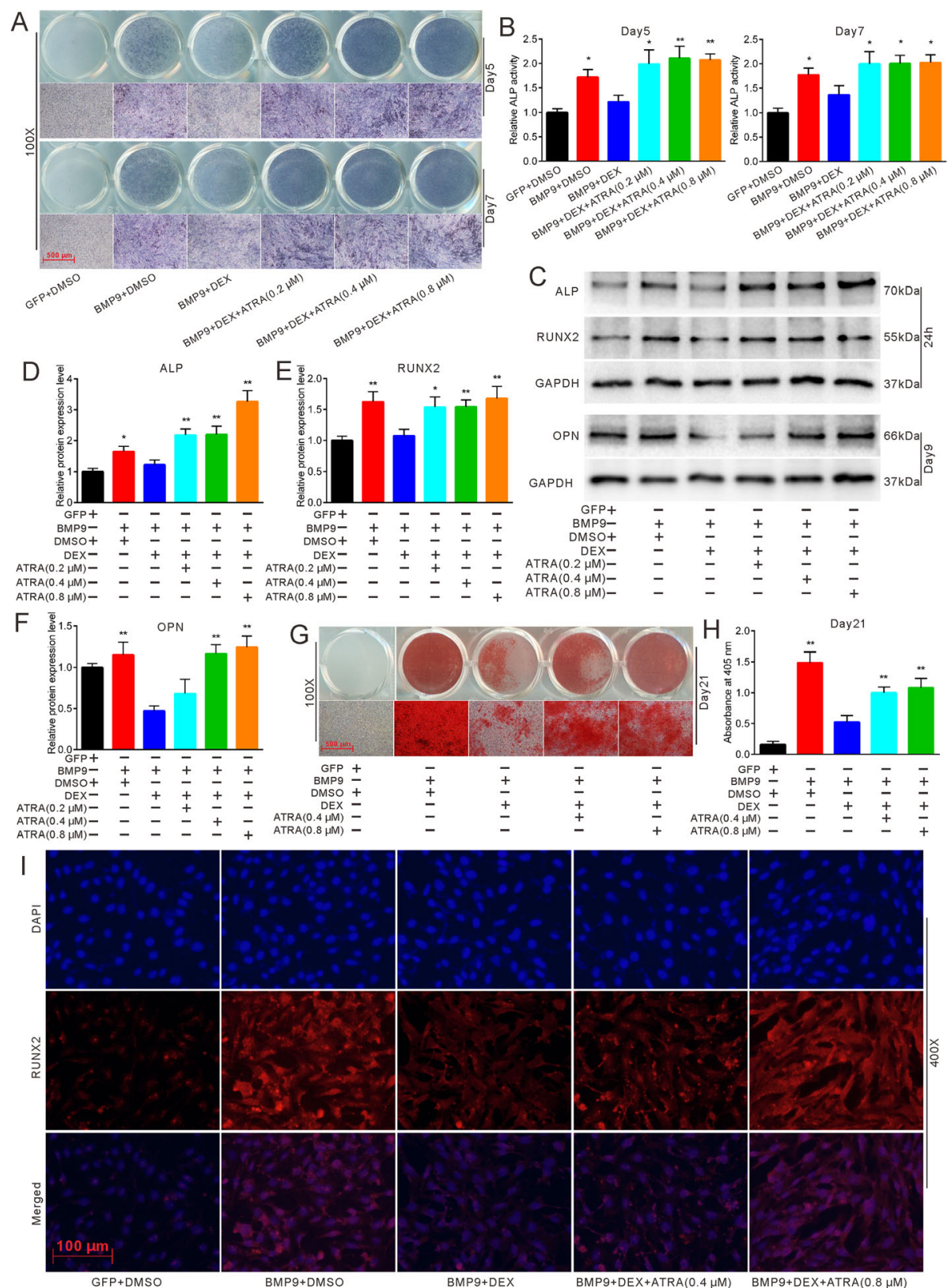

Fig. 3 Effects of ATRA on DEX-inhibited osteogenic differentiation. a ALP staining demonstrated the effect of different concentrations of ATRA on DEX-inhibited ALP activities in MEFs after 5 and 7 days. $\mathbf{b}$ Quantification of ALP staining showed the effect of different concentrations of ATRA on DEX-inhibited ALP activities, ${ }^{*} p<0.05$ vs BMP9 + DEX, ${ }^{* *} p<0.01$ vs BMP9 + DEX. $\mathbf{c}$ Western blot results demonstrated the effect of different concentrations of ATRA on DEX-inhibited protein expression of osteogenic markers. $\mathbf{d}-\mathbf{f}$ Quantification of western blot showed the effect of different concentrations of ATRA on DEX-inhibited osteogenic marker ALP, RUNX2, and OPN, ${ }^{*} p<0.05$ vs BMP9 + DEX, ${ }^{* *} p<0.01$ vs BMP9 + DEX. g Alizarin Red S staining showed the effect of different concentrations of ATRA on DEX-inhibited matrix mineralization in MEFs after 21 days. $\mathbf{h}$ Quantification of matrix mineralization demonstrated the effect of ATRA on DEX-inhibited matrix mineralization in MEFs, ${ }^{*} p<0.05$ vs BMP9 + DEX, ${ }^{* *} p<0.01$ vs BMP9 + DEX. i Immunofluorescent staining showed the effect of different concentrations of ATRA on DEX-inhibited RUNX2 expression after $24 \mathrm{~h}$. GFP, green fluorescent protein; DMSO, dimethyl sulfoxide; DEX, dexamethasone; OPN, osteopontin 

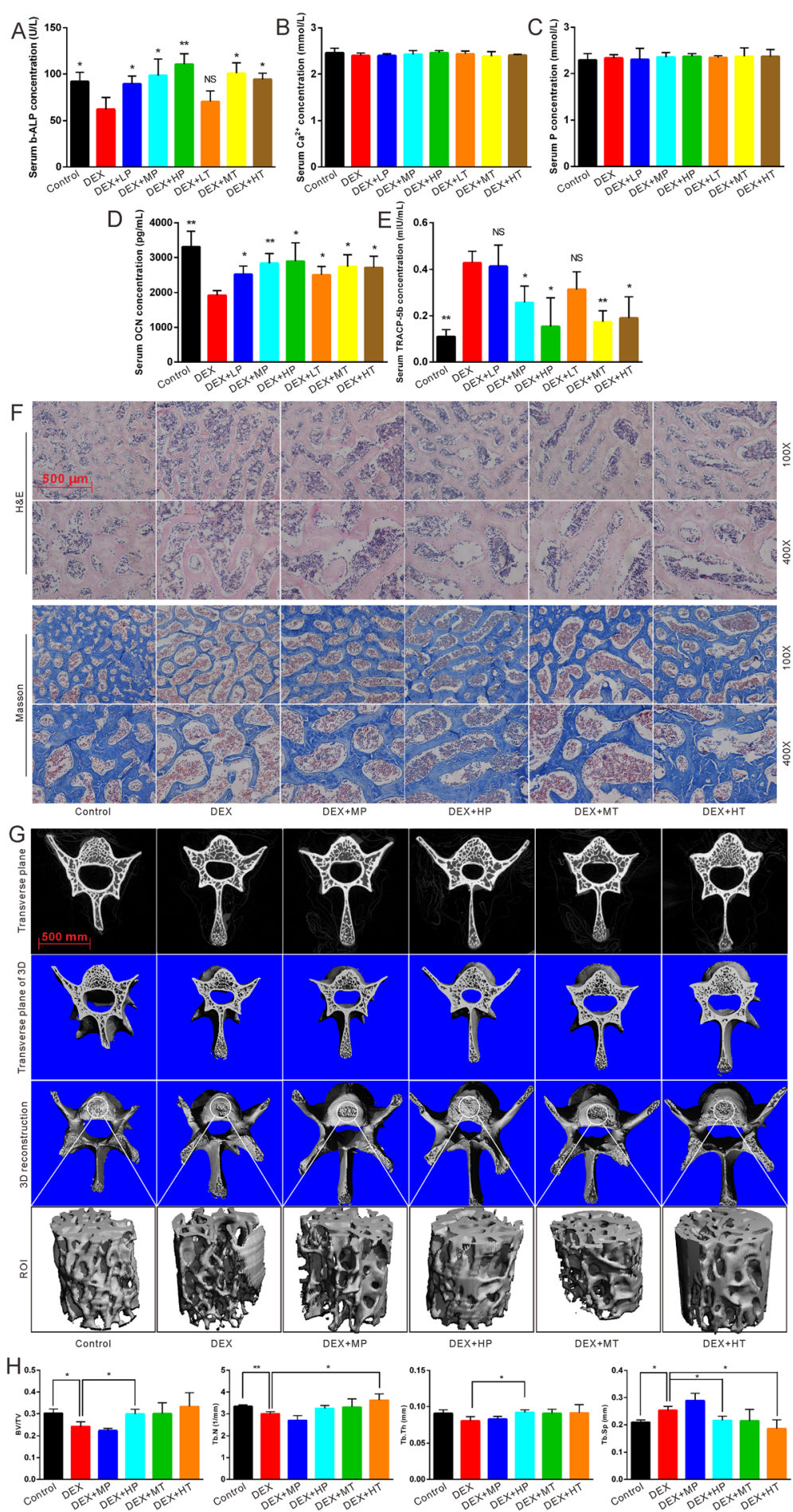

Fig. 4 Effects of ATRA on GIOP rat model in vivo. a-e Serum concentrations of ALP, calcium, phosphorus, OCN, and TRACP-5b in different groups, ${ }^{*} p<0.05$ vs DEX, ${ }^{* *} p<0.01$ vs DEX. $\mathbf{f}$ H\&E and Masson's trichrome staining showed the effect of ATRA on DEX-induced GIOP rats. $\mathbf{g} \mu \mathrm{CT}$ and three-dimensional reconstruction of vertebrae demonstrated the effect of ATRA on DEX-induced GIOP rats. $\mathbf{h}$ ROI analysis of vertebrae showed the effect of ATRA on DEX-induced GIOP rats, ${ }^{*} p<0.05,{ }^{* *} p<0.01$. OCN, osteocalcin; TRACP-5b, tartrate-resistant acid phosphatase $5 b$; $\mathrm{ROI}$, region of interest; BV/TV, ratio of bone volume to total volume; Tb.N, trabecular number; Tb.Th, trabecular thickness; Tb.Sp, trabecular separation; LP: prevention with low concentration of ATRA; MP: prevention with medium concentration of ATRA; HP: prevention with high concentration of ATRA; LT: treatment with low concentration of ATRA; MT: treatment with medium concentration of ATRA; HT: treatment with high concentration of ATRA 

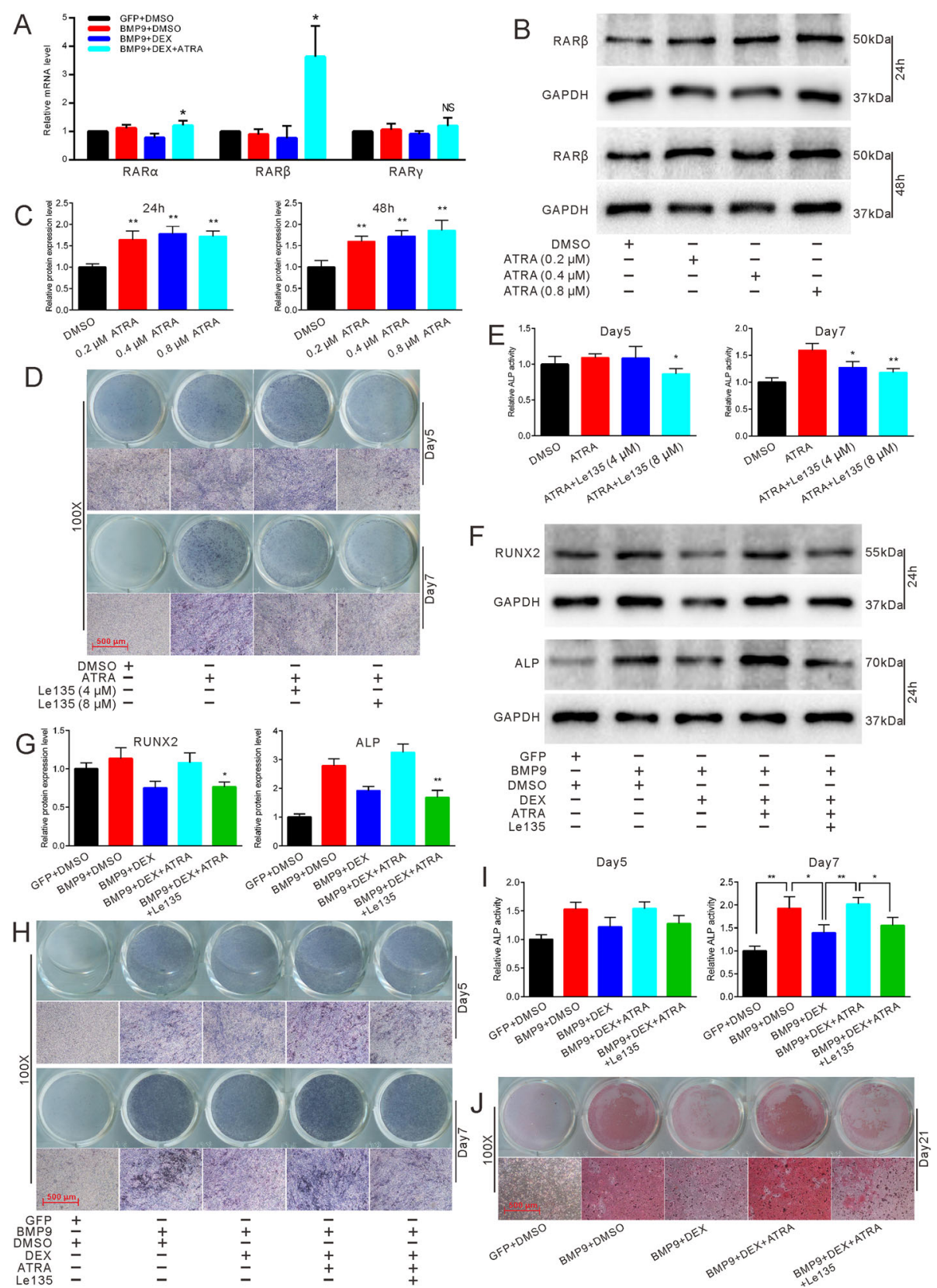

Fig. 5 Effects of RARß in the reversal process of ATRA in DEX-inhibited osteogenic differentiation. a RTgPCR results showed the effect of ATRA on mRNA expression of RARß in DEX-inhibited osteogenic differentiation in MEFs. $\mathbf{b}$ Western blot results showed the effect of different concentrations of ATRA on protein expression of RARB in MEFs. c Quantification of western blot showed the effect of ATRA on the expression of RAR $\beta$ in MEFs, ${ }^{*} p<0.05$ vs DMSO, ${ }^{*} p<0.01$ vs DMSO. d ALP staining demonstrated the effect of Le 135 on ATRA-induced ALP activities in MEFs after 5 and 7 days. e Quantification of ALP staining showed the effect of Le135 on ATRA-induced ALP activities, ${ }^{*} p<0.05$ vs ATRA, ${ }^{* *} p<0.01$ vs ATRA. $\mathbf{f}$ Western blot results showed the effect of Le135 on ATRA-reversed protein expression of osteogenic markers. $\mathbf{g}$ Quantification of western blot showed the effect of Le135 on ATRA-reversed osteogenic marker RUNX2 and ALP, ${ }^{*} p<0.05$ vs BMP9 + DEX + ATRA, ${ }^{* *} p<0.01$ vs BMP9 + DEX + ATRA. $\mathbf{h}$ ALP staining demonstrated the effect of Le135 on ATRA-reversed ALP activities in MEFs after 5 and 7 days. i Quantification of ALP staining showed the effect of Le135 on ATRA-reversed ALP activities, ${ }^{*} p<0.05$ vs BMP9 + DEX + ATRA, ${ }^{* *} p<0.01$ vs BMP9 + DEX + ATRA. $\mathbf{j}$ Alizarin Red S staining showed the effect of Le135 on ATRA-reversed matrix mineralization in MEFs after 21 days. GFP, green fluorescent protein; DMSO, dimethyl sulfoxide; DEX, dexamethasone; RAR $\beta$, retinoic acid receptor $\beta$; Le135, specific inhibitor of RARß 


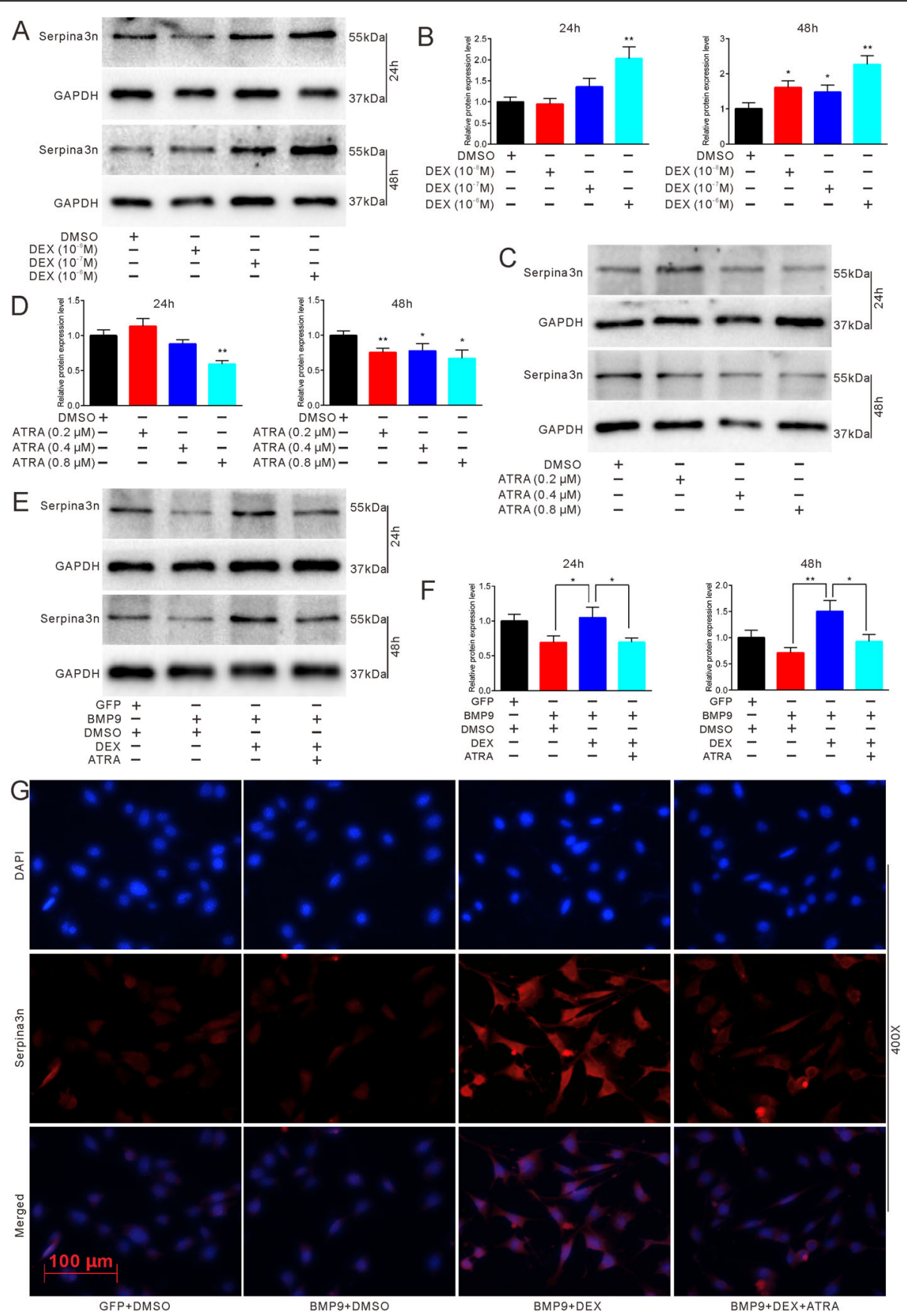

Fig. 6 Effects of ATRA and DEX on the expression of Serpina3n. a Western blot results showed the effect of different concentrations of DEX on protein expression of Serpina3n in MEFs. b Quantification of western blot showed the effect of different concentrations of DEX on the expression of Serpina3n in MEFs, ${ }^{*} p<0.05$ vs DMSO, ${ }^{* *} p<0.01$ vs DMSO. $\mathbf{c}$ Western blot results showed the effect of different concentrations of ATRA on protein expression of Serpina3n in MEFs. $\mathbf{d}$ Quantification of western blot showed the effect of different concentrations of ATRA on the expression of Serpina3n in MEFs, ${ }^{*} p<0.05$ vs DMSO, ${ }^{* *} p<0.01$ vs DMSO. e Western blot results showed the effect of ATRA and DEX on the expression of Serpina3n in BMP9-induced MEFs. $\mathbf{f}$ Quantification of western blot showed the effect of ATRA and DEX on the expression of Serpina3n in BMP9-induced MEFs, ${ }^{*} p<0.05,{ }^{* *} p<0.01$. g Immunofluorescent staining demonstrated the effect of ATRA and DEX on Serpina3n expression in BMP9-induced MEFs. GFP, green fluorescent protein; DMSO, dimethyl sulfoxide; DEX, dexamethasone 
involved in the reversal caused by ATRA with regard to DEX-inhibited osteogenesis.

\section{Effects of Serpina3n on the reversal process of ATRA with regard to DEX-inhibited osteogenesis}

Next, we sought to investigate the role of Serpina3n in the reversal caused by ATRA. We constructed adenovirusmediated overexpression and knockdown models of Serpina3n and validated their functions (Fig. 7a, b). ALP staining and quantification showed that the overexpression of Serpina3n can attenuate ATRA-induced ALP activities (Fig. 7c, d). In contrast, the knockdown of Serpina3n decreased the osteogenic inhibition of DEX with regard to BMP9 in MEFs (Fig. 7e, f). This result was confirmed by Western blot detection of the protein expressions of ALP and RUNX2 (Fig. $7 \mathrm{~g}, \mathrm{~h})$. Then, we combined the overexpression or knockdown of Serpina3n with DEX and ATRA in BMP9-induced osteogenic differentiation. ALP staining and quantification revealed that although the knockdown of Serpina3n hardly enhanced ATRA-reversed ALP activities, overexpression of Serpina3n significantly attenuated the ALP activities reversed by ATRA (Fig. 7i, j). These results indicate that DEX may inhibit BMP9-induced osteogenic differentiation by enhancing the expression of Serpina3n. In addition, ATRA may exert its reversal effect by decreasing Serpina3n.

\section{Effects of RAR $\beta$ on the expression of Serpina3n in the reversal process of ATRA with regard to DEX-inhibited osteogenesis}

Since RAR $\beta$ was found to be involved in the reversal process of ATRA, we recruited Le135 to further explore the relationship between ATRA and Serpina3n. RTqPCR results demonstrated that Le135 can promote the expression of Serpina3n and that this effect can be reversed when combined with ATRA (Fig. 8a). Western blot results and quantification showed that Le135 can enhance the expression of Serpina3n in a concentrationdependent manner with or without the combination of ATRA (Fig. 8b, c). Furthermore, ChIP results demonstrated that RAR $\beta$ can be added to the promoter region of Serpina3n to inhibit its transcription and expression either with or without the mediation of DEX and ATRA (Fig. 8d). The possible graphic mechanism showed that ATRA-activated RAR $\beta$ blocked the promotion of DEX (glucocorticoid) on Serpina3n to reverse the osteogenic inhibitory effect of DEX (Fig. 8e). These data allude that the reversal effect of ATRA on DEX-inhibited osteogenesis may be achieved by RAR $\beta$ activation and further Serpina3n inhibition.

\section{Discussion}

In this study, we found that ATRA facilitated BMP9induced or OIM-induced osteogenic differentiation in different MSC lines, while DEX exerted opposite effects.
Therefore, we used BMP9-induced MEFs as an osteogenic model to analyze the relationship between DEX and ATRA. We discerned that ATRA can reverse the inhibitory osteogenic effect of DEX in vitro as well as in vivo in the rat GIOP model. During the reversal process of ATRA, the expression of RAR $\beta$ was remarkably enhanced. In addition, Le135, the RAR $\beta$ specific inhibitor, blocked the reversal caused by ATRA, showing that ATRA may exert its function through RAR $\beta$. Furthermore, RNA-seq revealed that Serpina3n, the novel gene, was upregulated by DEX but downregulated by ATRA. Meanwhile, knockdown of Serpina3n also reversed DEX-inhibited osteogenic differentiation to some extent, indicating that the reversal effect of ATRA may be achieved by Serpina3n inhibition. ChIP analysis confirmed that RAR $\beta$ can bind to the promoter region of Serpina3n. Hence, ATRA may reverse DEX-inhibited osteogenic differentiation via RAR $\beta$ activation and the subsequent inhibition of Serpina3n transcription.

DEX, a synthetic glucocorticoid, has been widely used in the treatment of inflammatory and autoimmune diseases such as systemic lupus erythematosus (SLE), allergies, and rheumatoid arthritis (RA). However, high dosage and long-term use of DEX are associated with severe complications such as GIOP and osteonecrosis of the femoral head (ONFH). Previous studies have asserted that DEX has a dual role in osteogenic differentiation [44]. DEX promotes osteogenesis at the physiological concentration but dramatically inhibits bone metabolism at the pharmacological concentration. Thus, a low concentration of DEX $\left(10^{-8} \mathrm{M}\right)$ is routinely used in OIM. In our study, the osteogenic potential of DEX was higher at a concentration of $10^{-9} \mathrm{M}$ than at $10^{-8} \mathrm{M}$ (Fig. 2i-l). This observation could be probably because the optimal concentration of DEX that facilitates osteogenic differentiation differs from cell to cell. Our results also proved that a low concentration of DEX enhances osteogenesis while a high concentration exerts the opposite effect (Fig. 2c-h). The effects of DEX on bone metabolism are manifested as osteoblastic inhibition and osteoclastic promotion. DEX induces the apoptosis of osteoblasts and osteocytes by binding to monomeric glucocorticoid receptor (GR); meanwhile, the osteoclasts are activated, leading to an increase in the RANKL/OPG ratio. In addition, adipogenesis is markedly enhanced by DEX in the mesenchymal progenitor cells to compensate the bone loss with adipocytes [2, 44, 45]. Our study showed that DEX decreased the number and thickness of the trabecular bone but increased trabecular separation, which is consistent with previous reports.

Osteogenesis-related studies on ATRA, a derivative of vitamin A, remain controversial. Several studies have reported that ATRA inhibits osteogenic differentiation [46, 47]. However, an increasing number of studies have 

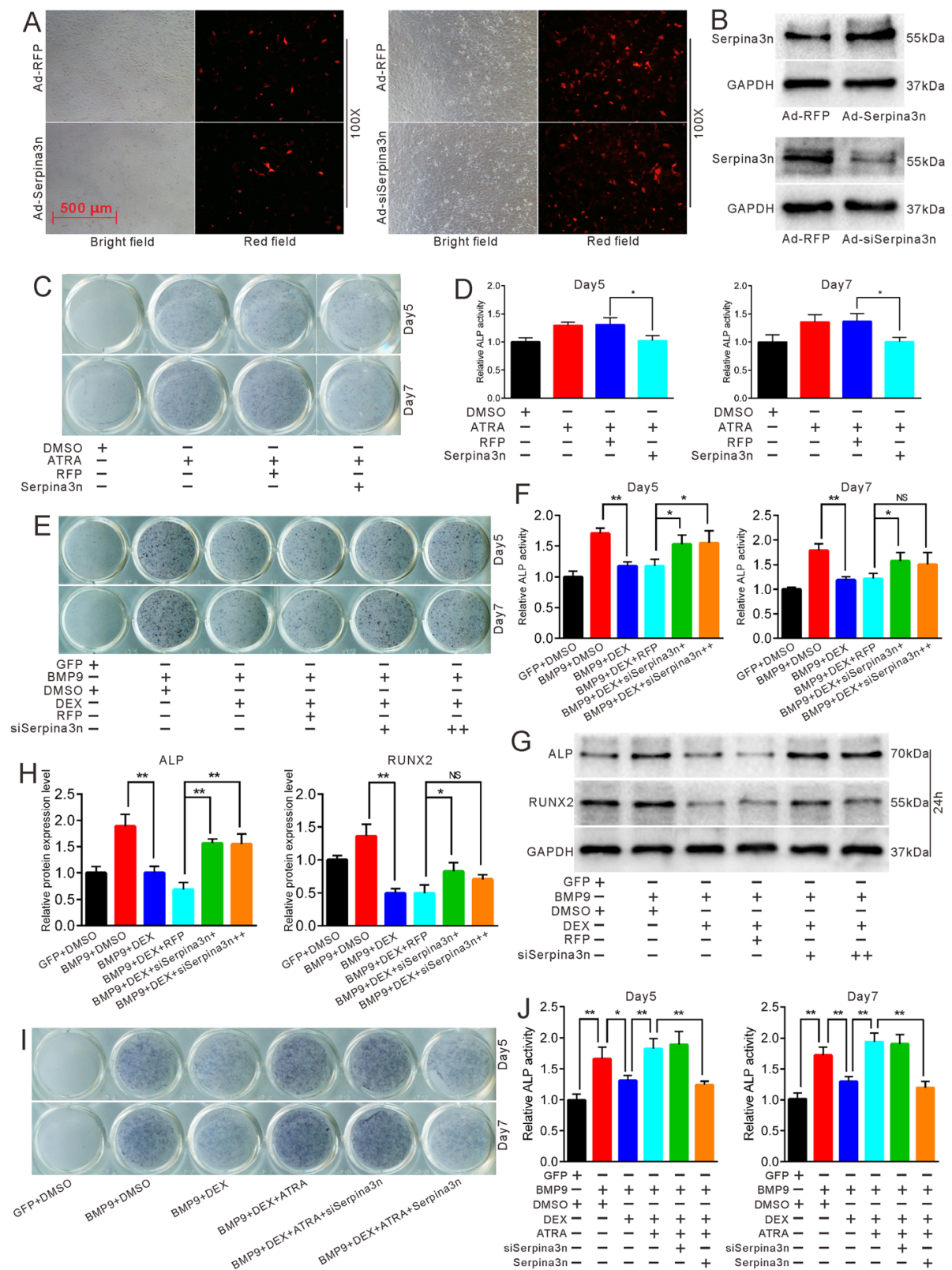

Fig. 7 Effects of Serpina3n overexpression and knockdown on the reversal process of ATRA in DEX-inhibited osteogenic differentiation. a MEFs infected with adenovirus-mediated Ad-RFP, Ad-siSerpina3n, and Ad-Serpina3n after $24 \mathrm{~h}$. b Western blot showed the protein expression of Serpina3n in MEFs $24 \mathrm{~h}$ after Ad-RFP, Ad-siSerpina3n and Ad-Serpina3n infection. c ALP staining demonstrated the effect of overexpression of Serpina3n on ATRA-induced ALP activities in MEFs after 5 and 7 days. $\mathbf{d}$ Quantification of ALP staining showed the effect of overexpression of Serpina3n on ATRA-induced ALP activities, ${ }^{*} p<0.05,{ }^{* *} p<0.01$. e ALP staining showed the effect of knockdown of Serpina3n on DEX-inhibited ALP activities induced by BMP9. $\mathbf{f}$ Quantification of ALP staining showed the effect of knockdown of Serpina3n on DEX-inhibited ALP activities, ${ }^{*} p$ $<0.05$, ${ }^{* *} p<0.01$. $\mathbf{g}$ Western blot results showed the effect of knockdown of Serpina3n on DEX-inhibited osteogenic marker ALP and RUNX2 induced by BMP9 in MEFs. $\mathbf{h}$ Quantification of western blot demonstrated the effect of knockdown of Serpina3n on DEX-inhibited osteogenic markers in MEFs, ${ }^{*} p<0.05,{ }^{* *} p<0.01$. i ALP staining showed the effect of Serpina3n overexpression and knockdown on ATRA-reversed ALP activities in MEFs. $\mathbf{j}$ Quantification of ALP staining showed the effect of Serpina3n on ATRA-reversed ALP activities, ${ }^{*} p<0.05,{ }^{* *} p<0.01$. GFP, green fluorescent protein; RFP, red fluorescent protein; DMSO, dimethyl sulfoxide; DEX, dexamethasone 


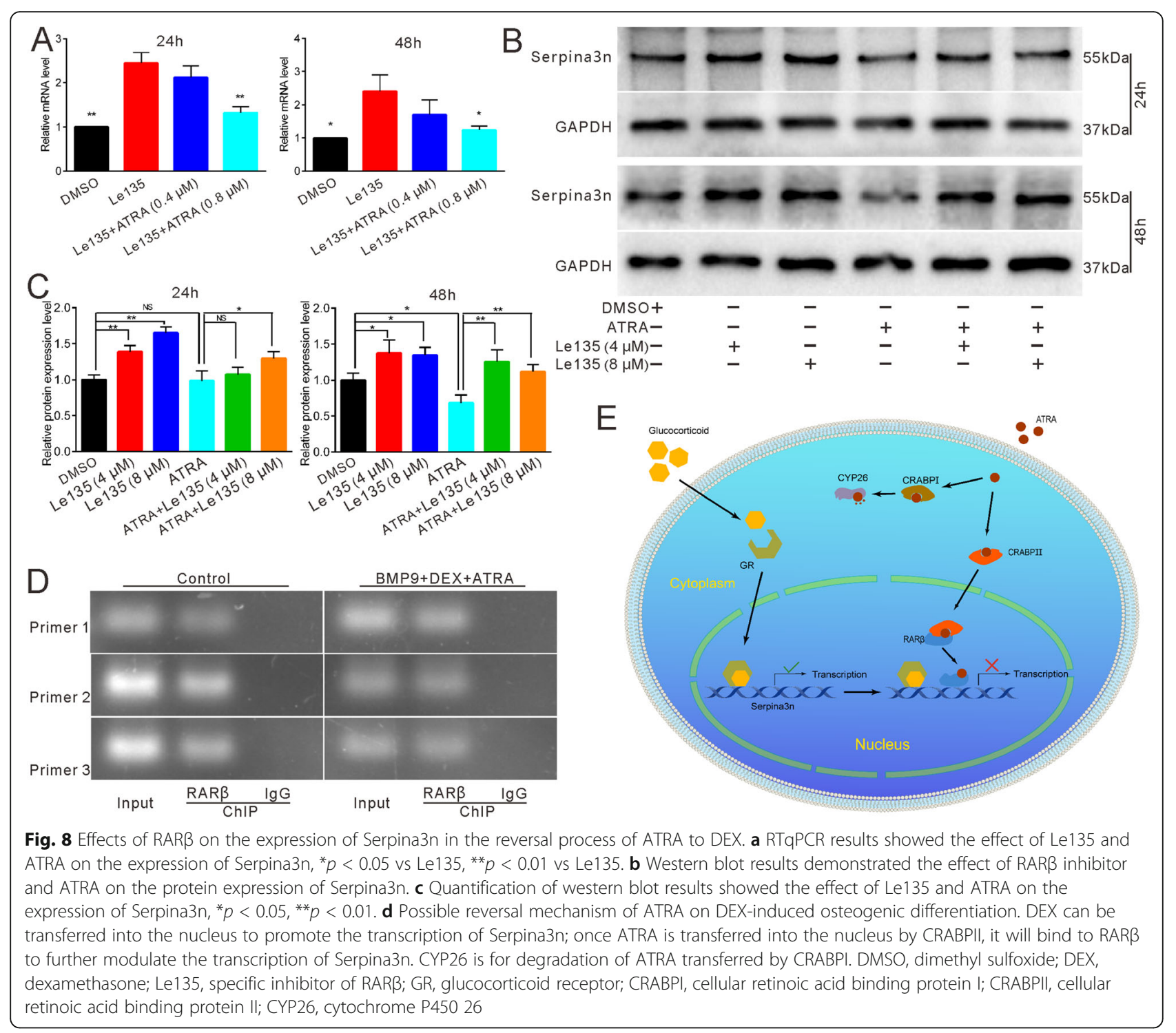

revealed the effect of ATRA on osteogenic differentiation. Previous studies have established that ATRA can increase the osteogenic ability of BMP9 [32, 43]. Moreover, ATRA also engages in a crosstalk with Wnt/ $\beta$-catenin to potentiate Wnt3A-induced osteogenic differentiation $[43,48]$. Our study showed that ATRA can not only stimulate BMP9-induced osteogenic differentiation (Fig. 1g-k) but also enhance OIM-induced osteogenesis (Fig. 1l, m). However, on its own, ATRA only induced ALP activities but not matrix mineralization in MSCs (Fig. 1a-d and Fig. S1A-B), which agrees with a previous study [49]. Nevertheless, no studies have so far reported the varying effects of ATRA alone on early and late osteogenic markers; the mechanism remains unknown and awaits further investigations. It has been reported that ATRA can bind to RARs and RXRs. However, RXRs are restrictively activated when the concentration of ATRA is higher than $10^{-5} \mathrm{M}$ [29]. As receptors of ATRA, RARs play an important role in bone metabolism. RAR $\gamma$ directly regulates endochondral ossification and indirectly regulates the formation of tibial vessels [50]. RAR agonists attenuate RANKL-mediated osteoclastic differentiation [51]. Besides, RAR $\alpha$ acts as an important regulatory component in the differentiation of the mesoderm into chondroblasts [52]. The investigation of RAR $\beta$ in osteogenic differentiation seems rare. Our study demonstrated that the expression of RAR $\beta$ was significantly elevated by ATRA in the DEXinhibited osteogenic model (Fig. 5a) and that Le135 blocked the reversal of ATRA (Fig. $5 \mathrm{f}-\mathrm{j}$ ). Therefore, RAR $\beta$ may be a crucial receptor in the osteogenic reversal process of ATRA.

Being a novel gene, studies on Serpina3n are limited. Most of the investigations are related to neurology 
because Serpina3n also acts as a reactive astroglial marker. It has been reported that DEX can remarkably enhance both the gene and protein levels of Serpina3n and that Serpina3n may act as a circulating biomarker for muscle atrophy induced by glucocorticoids [37, 53]. In our study, DEX potentiated the expression of Serpina3 $\mathrm{n}$ in a concentration-dependent manner (Fig. 6a, b), which is consistent with our RNA-seq results and previous reports involving different cell lines. However, the relationship between Serpina3n and ATRA has not been well explored although ATRA has been shown to be brain protective in a traumatic brain model by partly attenuating the expression of Serpina3n [54]. In our study, ATRA reduced the expression of Serpina3n with or without the mediation of DEX (Fig. 5c, d-g). Furthermore, overexpression of Serpina3n attenuated ATRAinduced ALP activities (Fig. 7c, d), whereas knockdown of Serpina3n partly blocked DEX-inhibited osteogenic differentiation (Fig. 7e, f). Thus, when combined with RAR $\beta$, our data strongly suggest that DEX may inhibit BMP9-induced or OIM-induced osteogenic differentiation by enhancing Serpina3n and that ATRA reverses DEX-inhibited osteogenesis by targeting Serpina3n, which is mediated by RAR $\beta$.

In our study, we utilized Le135 to explore the role of RAR $\beta$. Although the results are convincing, RAR $\beta$ overexpression or agonists were not introduced or applied in our analysis. This investigation needs to be performed in the future to validate our present results. Furthermore, even though we have investigated the in vitro expressions of Serpina3n during the mediation of DEX and ATRA, the in vivo expressions of Serpina3n and RAR $\beta$ remain to be tested. In further studies, we plan to focus on Serpina3n using GIOP and ovariectomy models to further explore the role of Serpina3n in GIOP and other osteoporosis. Furthermore, the effects of ATRA on matrix mineralization were inconsistent when compared with the ALP activities induced by ATRA, which requires further investigations.

\section{Conclusion}

Collectively, our findings suggest that ATRA can reverse the inhibitory osteogenic effect of DEX both in vitro and in vivo. ATRA may chiefly activate RAR $\beta$ and subsequently inhibit the transcription of Serpina3n to antagonize DEX-inhibited osteogenic differentiation. Our results are expected to provide useful insights in the journey of discovering new agents and drugs as well as in identifying the key genes involved in GIOP.

\section{Abbreviations}

ATRA: All-trans retinoic acid; DEX: Dexamethasone; Serpina3n: Serine protease inhibitor 3n; MSCs: Mesenchymal stem cells; RARB: Retinoic acid receptor $\beta$; GFP: Green fluorescent protein; RFP: Red fluorescent protein; BMP9: Bone morphogenetic protein 9; ALP: Alkaline phosphatase;
RUNX2: Runt-related transcription factor 2; OPN: Osteopontin; OCN: Osteocalcin; DMSO: Dimethyl sulfoxide; DMEM: Dulbecco's modified Eagle's medium; OIM: Osteogenic inductive medium; GR: Glucocorticoid receptor; CRABPI: Cellular retinoic acid binding protein I; CRABPII: Cellular retinoic acid binding protein II; CYP26: Cytochrome P450 26; OVX: Ovariectomy

\section{Supplementary Information}

The online version contains supplementary material available at https://doi. org/10.1186/s13287-021-02347-0.

Additional file 1: Fig. S1. (A) Alizarin Red S staining showed the effect of different concentrations of ATRA on matrix mineralization in MEFs after 14 days. (B) Quantification of matrix mineralization in MEFs after 14 days, ${ }^{*} p<0.05$ vs DMSO, ${ }^{* *} p<0.01$ vs DMSO. (C) ALP staining showed the effect of different concentrations of ATRA on BMP9-induced ALP activities in $\mathrm{C} 3 \mathrm{H} 10 \mathrm{~T} 1 / 2$ cells after 5 and 7 days. (D-E) Quantification of ALP staining showed the effect of different concentrations of ATRA on BMP9-induced ALP activities in C3H10T1/2 cells after 5 and 7 days, ${ }^{*} p<0.05$ vs BMP9 + DMSO, ${ }^{* *} \mathrm{p}<0.01$ vs BMP9 + DMSO. (F) ALP staining showed the effect of different concentrations of ATRA on BMP9-induced ALP activities in C2C12 cells after 5 and 7 days. (G-H) Quantification of ALP staining showed the effect of different concentrations of ATRA on BMP9-induced ALP activities in C2C12 cells after 5 and 7 days, ${ }^{*} p<0.05$ vs BMP9 + DMSO, ${ }^{* *} p<0.01$ vs BMP9 + DMSO. (I-J) Alizarin Red S staining and quantification showed the effect of different concentrations of ATRA on BMP9-induced matrix mineralization in MEFs after 21 days, ${ }^{*} p<0.05$ vs BMP9 + DMSO, ${ }^{* *} \mathrm{p}<0.01$ vs BMP9 + DMSO.

Additional file 2: Fig. S2. (A) ALP staining showed the effect of different concentrations of DEX on BMP9-induced ALP activities in C3H10T1/2 cells after 5 and 7 days. (B-C) Quantification of ALP staining showed the effect of different concentrations of DEX on BMP9-induced ALP activities in C3H10T1/2 cells after 5 and 7 days, ${ }^{*} p<0.05$ vs BMP9 + DMSO, ${ }^{* *} p<0.01$ vs BMP9 + DMSO. (D) ALP staining showed the effect of different concentrations of DEX on BMP9-induced ALP activities in C2C12 cells after 5 and 7 days. (E-F) Quantification of ALP staining showed the effect of different concentrations of DEX on BMP9-induced ALP activities in C2C12 cells after 5 and 7 days, ${ }^{*} p<0.05$ vs BMP9 + DMSO, ${ }^{* *} \mathrm{p}<0.01$ vs BMP9 + DMSO.

Additional file 3: Fig. S3. (A) ALP staining showed the effect of different concentrations of ATRA on different concentrations of DEX-inhibited ALP aCtivities in C3H10T1/2 cells after 5 days. (B-E) Quantification of ALP staining showed the effect of different concentrations of ATRA on different concentrations of DEX-inhibited ALP activities, ${ }^{*} \mathrm{p}<0.05,{ }^{* *} \mathrm{p}<0.01$.

\section{Acknowledgements}

We would like to thank professor Tong-Chuan He (University of Chicago Medical Center) sincerely for the generous provision of recombinant adenoviruses used in this study; the authors would like to thank Mr Kenley Mew (Chongqing Medical University) for his help with grammar mistake revision.

\section{Authors' contributions}

ZLD and HTJ designed this study. HTJ and RD carried out most of the experiments and data analysis. YD, YXD, YYY, and HHL helped with animal model and in vivo sample harvest. CCR and NN helped with some in vitro experiments and statistical analysis. MN helped with paper editing and format revision. The final manuscript was approved by all authors.

\section{Funding}

This work was supported by research grant from the National Natural Science Foundation of China (NSFC), grant number 81672230 to ZhongLiang Deng; research grant from the Natural Science Foundation of Chongqing, grant number cstc2019jscx-gksbX0027 to Zhong-Liang Deng and cstc2019jcyj-msxmX0249 to Mao Nie.

Availability of data and materials

All datasets used and analyzed in study are available from the corresponding author on reasonable request. 


\section{Declarations}

\section{Ethics approval and consent to participate}

For animal experiments, this study was approved by the Ethics Committee of Chongqing Medical University (CQMU), including the rat euthanasia procedure.

\section{Consent for publication}

Not applicable.

\section{Competing interests}

All authors declare that they have no competing interests.

\section{Author details}

'Department of Orthopaedics, The Second Affiliated Hospital of Chongqing Medical University, 76 Linjiang Road, Yuzhong District, Chongqing 400010, China. ${ }^{2}$ Chongqing Key Laboratory of Biochemistry and Molecular Pharmacology, Chongqing Medical University, No. 1 Yixueyuan Road, Yuzhong District, Chongqing 400010, China. ${ }^{3}$ Department of Pharmacology, School of Pharmacy, Chongqing Medical University, No. 1 Yixueyuan Road, Yuzhong District, Chongqing 400010, China. ${ }^{4}$ Ministry of Education Key Laboratory of Diagnostic Medicine, School of Laboratory Medicine, Chongqing Medical University, No. 1 Yixueyuan Road, Yuzhong District, Chongqing 400010, China.

\section{Received: 20 October 2020 Accepted: 19 April 2021}

\section{Published online: 17 May 2021}

\section{References}

1. Buckley L, Humphrey M. Glucocorticoid-induced osteoporosis. N Engl J Med. 2018;379(26):2547-56. https://doi.org/10.1056/NEJMcp1800214.

2. Chotiyarnwong P, McCloskey E. Pathogenesis of glucocorticoid-induced osteoporosis and options for treatment. Nat Rev Endocrinol. 2020;16(8):43747. https://doi.org/10.1038/s41574-020-0341-0.

3. Compston J. Glucocorticoid-induced osteoporosis: an update. Endocrine. 2018:61(1):7-16. https://doi.org/10.1007/s12020-018-1588-2.

4. Briot K, Roux C. Glucocorticoid-induced osteoporosis. RMD Open. 2015;1(1): e000014. https://doi.org/10.1136/rmdopen-2014-000014.

5. Weinstein R, Jilka R, Parfitt A, Manolagas S. Inhibition of osteoblastogenesis and promotion of apoptosis of osteoblasts and osteocytes by glucocorticoids. Potential mechanisms of their deleterious effects on bone. J Clin Invest. 1998;102(2):274-82. https://doi.org/10.1172/JCI2799.

6. Hofbauer L, Rauner M. Minireview: live and let die: molecular effects of glucocorticoids on bone cells. Mol Endocrinol (Baltimore, Md). 2009;23(10): 1525-31.

7. Kim H, Zhao H, Kitaura H, Bhattacharyya S, Brewer J, Muglia L, et al. Glucocorticoids suppress bone formation via the osteoclast. J Clin Invest. 2006;116(8):2152-60. https://doi.org/10.1172/JCI28084.

8. Jia D, O'Brien C, Stewart S, Manolagas S, Weinstein R. Glucocorticoids act directly on osteoclasts to increase their life span and reduce bone density. Endocrinology. 2006;147(12):5592-9. https://doi.org/10.1210/en.2006-0459.

9. Teitelbaum S. Glucocorticoids and the osteoclast. Clin Exp Rheumatol. 2015; 33(4 Suppl 92):S37-9.

10. Wu Z, Bucher N, Farmer S. Induction of peroxisome proliferator-activated receptor gamma during the conversion of $3 \mathrm{~T} 3$ fibroblasts into adipocytes is mediated by C/EBPbeta, C/EBPdelta, and glucocorticoids. Mol Cell Biol. 1996;16(8):4128-36. https://doi.org/10.1128/MCB.16.8.4128.

11. Yang Y, Zhu Z, Wang D, Zhang X, Liu Y, Lai W, et al. Tanshinol alleviates impaired bone formation by inhibiting adipogenesis via KLF15/PPARY2 signaling in GlO rats. Acta Pharmacol Sin. 2018;39(4):633-41. https://doi. org/10.1038/aps.2017.134

12. Cosman F, de Beur S, LeBoff M, Lewiecki E, Tanner B, Randall S, et al. Clinician's guide to prevention and treatment of osteoporosis. Osteoporos Int. 2014;25(10):2359-81. https://doi.org/10.1007/s00198-014-2794-2.

13. Luu H, Song W, Luo X, Manning D, Luo J, Deng Z, et al. Distinct roles of bone morphogenetic proteins in osteogenic differentiation of mesenchymal stem cells. J Orthop Res. 2007;25(5):665-77. https://doi. org/10.1002/jor.20359

14. Chen D, Zhao M, Mundy G. Bone morphogenetic proteins. Growth Factors (Chur, Switzerland). 2004;22(4):233-41.
15. Sharff K, Song W, Luo X, Tang N, Luo J, Chen J, et al. Hey1 basic helix-loophelix protein plays an important role in mediating BMP9-induced osteogenic differentiation of mesenchymal progenitor cells. J Biol Chem. 2009:284(1):649-59. https://doi.org/10.1074/jbc.M806389200.

16. Chen L, Jiang W, Huang J, He B, Zuo G, Zhang W, et al. Insulin-like growth factor 2 (IGF-2) potentiates BMP-9-induced osteogenic differentiation and bone formation. J Bone Miner Res. 2010;25(11):2447-59. https://doi.org/10.1 002/jbmr.133.

17. Chen L, Zou X, Zhang R, Pi C, Wu N, Yin L, et al. IGF1 potentiates BMP9induced osteogenic differentiation in mesenchymal stem cells through the enhancement of BMP/Smad signaling. BMB Rep. 2016;49(2):122-7. https:// doi.org/10.5483/BMBRep.2016.49.2.228.

18. Liao $Y$, Du W, Hu Y, Li F, Ma Y, Wang H, et al. CREB/Wnt10b mediates the effect of COX-2 on promoting BMP9-induced osteogenic differentiation via reducing adipogenic differentiation in mesenchymal stem cells. J Cell Biochem. 2019;120(6):9572-87. https://doi.org/10.1002/jcb.28234.

19. Wang J, Liu Y, Yin L, Chen L, Huang J, Liu Y, et al. BMP9 and COX-2 form an important regulatory loop in BMP9-induced osteogenic differentiation of mesenchymal stem cells. Bone. 2013;57(1):311-21. https://doi.org/10.1016/j. bone.2013.08.015

20. Huang J, Yuan S, Wang D, Wu Q, Wang X, Pi C, et al. The role of COX-2 in mediating the effect of PTEN on BMP9 induced osteogenic differentiation in mouse embryonic fibroblasts. Biomaterials. 2014;35(36):9649-59. https:// doi.org/10.1016/j.biomaterials.2014.08.016.

21. Zhang H, Wang J, Deng F, Huang E, Yan Z, Wang Z, et al. Canonical Wnt signaling acts synergistically on BMP9-induced osteo/odontoblastic differentiation of stem cells of dental apical papilla (SCAPs). Biomaterials. 2015;39:145-54. https://doi.org/10.1016/j.biomaterials.2014.11.007.

22. Lin L, Qiu Q, Zhou N, Dong W, Shen J, Jiang W, et al. Dickkopf-1 is involved in BMP9-induced osteoblast differentiation of $\mathrm{C} 3 \mathrm{H} 10 \mathrm{~T} 1 / 2$ mesenchymal stem cells. BMB Rep. 2016;49(3):179-84. https://doi.org/10.5483/BMBRep.201 6.49.3.206.

23. Song B, Li X, Yao Y, Xu Q, Meng X, Huang C, et al. BMP9 inhibits the proliferation and migration of fibroblast-like synoviocytes in rheumatoid arthritis via the PI3K/AKT signaling pathway. Int Immunopharmacol. 2019;74: 105685. https://doi.org/10.1016/j.intimp.2019.105685.

24. Li S, Dai H, He Y, Peng S, Zhu T, Wu Y, et al. BMP9 inhibits the growth of breast cancer cells by downregulation of the PI3K/Akt signaling pathway. Oncol Rep. 2018;40(3):1743-51. https://doi.org/10.3892/or.2018.6572.

25. Porcù E, Maule F, Boso D, Rampazzo E, Barbieri V, Zuccolotto G, et al. BMP9 counteracts the tumorigenic and pro-angiogenic potential of glioblastoma. Cell Death Differ. 2018;25(10):1808-22. https://doi.org/10.1 038/s41418-018-0149-9.

26. Costantini L, Molinari R, Farinon B, Merendino N. Retinoic acids in the treatment of most lethal solid cancers. J Clin Med. 2020;9(2):360. https://doi. org/10.3390/jcm9020360.

27. di Masi A, Leboffe L, De Marinis E, Pagano F, Cicconi L, Rochette-Egly $C$, et al. Retinoic acid receptors: from molecular mechanisms to cancer therapy. Mol Asp Med. 2015;41:1-115. https://doi.org/10.1016/j.mam.2 014.12.003.

28. Bushue N, Wan Y. Retinoid pathway and cancer therapeutics. Adv Drug Deliv Rev. 2010;62(13):1285-98. https://doi.org/10.1016/j.addr.2010.07.003.

29. Heyman RA, Mangelsdorf DJ, Dyck JA, Stein RB, Eichele G, Evans RM, et al. 9 -cis retinoic acid is a high affinity ligand for the retinoid $X$ receptor. Cell. 1992;68(2):397-406. https://doi.org/10.1016/0092-8674(92)90479-V.

30. Larange $\mathrm{A}$, Cheroutre $\mathrm{H}$. Retinoic acid and retinoic acid receptors as pleiotropic modulators of the immune system. Annu Rev Immunol. 2016; 34(1):369-94. https://doi.org/10.1146/annurev-immunol-041015-055427.

31. Shao $Y$, Chen $Q$, Zeng $Y$, Li Y, Ren W, Zhou L, et al. All-trans retinoic acid shifts rosiglitazone-induced adipogenic differentiation to osteogenic differentiation in mouse embryonic fibroblasts. Int J Mol Med. 2016;38(6): 1693-702. https://doi.org/10.3892/ijmm.2016.2782.

32. Liu Y, Liu Y, Zhang R, Wang X, Huang F, Yan Z, et al. All-trans retinoic acid modulates bone morphogenic protein 9-induced osteogenesis and adipogenesis of preadipocytes through BMP/Smad and Wnt/B-catenin signaling pathways. Int J Biochem Cell Biol. 2014;47:47-56. https://doi.org/1 0.1016/j.biocel.2013.11.018

33. Heit C, Jackson B, McAndrews M, Wright M, Thompson D, Silverman G, et al. Update of the human and mouse SERPIN gene superfamily. Hum Genomics. 2013;7(1):22. https://doi.org/10.1186/1479-7364-7-22. 
34. Horvath A, Forsyth S, Coughlin P. Expression patterns of murine antichymotrypsin-like genes reflect evolutionary divergence at the Serpina3 locus. J Mol Evol. 2004;59(4):488-97. https://doi.org/10.1007/ s00239-004-2640-9.

35. Hsu I, Parkinson L, Shen Y, Toro A, Brown T, Zhao H, et al. Serpina3n accelerates tissue repair in a diabetic mouse model of delayed wound healing. Cell Death Dis. 2014;5(10):e1458. https://doi.org/10.1038/cddis.2 014.423 .

36. Gong G, Song S, Xu X, Luo Q, Han Q, He J, et al. Serpina3n is closely associated with fibrotic procession and knockdown ameliorates bleomycininduced pulmonary fibrosis. Biochem Biophys Res Commun. 2020;532(4): 598-604. https://doi.org/10.1016/j.bbrc.2020.08.094.

37. Gueugneau M, d'Hose D, Barbé C, de Barsy M, Lause P, Maiter D, et al. Increased Serpina3n release into circulation during glucocorticoid-mediated muscle atrophy. J Cachexia Sarcopenia Muscle. 2018;9(5):929-46. https://doi. org/10.1002/jcsm.12315.

38. Ishida M, Kawao N, Okada K, Tatsumi K, Sakai K, Nishio K, et al. Serpina3n, dominantly expressed in female osteoblasts, suppresses the phenotypes of differentiated osteoblasts in mice. Endocrinology. 2018;159(11):3775-90. https://doi.org/10.1210/en.2018-00639.

39. He TC, Zhou S, da Costa LT, Yu J, Kinzler KW, Vogelstein B. A simplified system for generating recombinant adenoviruses. Proc Natl Acad Sci U S A. 1998:95(5):2509-14. https://doi.org/10.1073/pnas.95.5.2509.

40. Luo J, Deng ZL, Luo X, Tang N, Song WX, Chen J, et al. A protocol for rapid generation of recombinant adenoviruses using the AdEasy system. Nat Protoc. 2007;2(5):1236-47. https://doi.org/10.1038/nprot.2007.135.

41. Jiang $H$, Ran C, Liao $Y$, Zhu J, Wang H, Deng R, et al. IGF-1 reverses the osteogenic inhibitory effect of dexamethasone on BMP9-induced osteogenic differentiation in mouse embryonic fibroblasts via PI3K/AKT/ COX-2 pathway. J Steroid Biochem Mol Biol. 2019;191:105363. https://doi. org/10.1016/j.jsbmb.2019.04.012.

42. Lengner C, Lepper C, van Wijnen A, Stein J, Stein G, Lian J. Primary mouse embryonic fibroblasts: a model of mesenchymal cartilage formation. J Cell Physiol. 2004;200(3):327-33. https://doi.org/10.1002/jcp.20118.

43. Wang $H, H u$ Y, He F, Li L, Li P, Deng Y, et al. All-trans retinoic acid and COX2 cross-talk to regulate BMP9-induced osteogenic differentiation via Wnt/Bcatenin in mesenchymal stem cells. Biomed Pharmacother. 2019;118:109279.

44. Seibel M, Cooper M, Zhou H. Glucocorticoid-induced osteoporosis: mechanisms, management, and future perspectives. Lancet Diabetes Endocrinol. 2013;1(1):59-70. https://doi.org/10.1016/S2213-8587(13)70045-7.

45. Rauch A, Seitz S, Baschant U, Schilling A, Illing A, Stride B, et al. Glucocorticoids suppress bone formation by attenuating osteoblast differentiation via the monomeric glucocorticoid receptor. Cell Metab. 2010; 11(6):517-31. https://doi.org/10.1016/j.cmet.2010.05.005.

46. Liu Y, Ma X, Guo J, Lin Z, Zhou M, Bi W, et al. All-trans retinoic acid can antagonize osteoblastogenesis induced by different BMPs irrespective of their dimerization types and dose-efficiencies. Drug Design Dev Ther. 2018; 12:3419-30. https://doi.org/10.2147/DDDT.S178190.

47. Green A, Kocovski P, Jovic T, Walia M, Chandraratna R, Martin T, et al. Retinoic acid receptor signalling directly regulates osteoblast and adipocyte differentiation from mesenchymal progenitor cells. Exp Cell Res. 2017;350(1): 284-97. https://doi.org/10.1016/j.yexcr.2016.12.007.

48. Zhang S, Chen X, Hu Y, Wu J, Cao Q, Chen S, et al. All-trans retinoic acid modulates Wnt3A-induced osteogenic differentiation of mesenchymal stem cells via activating the PI3KAKT/GSK3 3 signalling pathway. Mol Cell Endocrinol. 2016;422:243-53. https://doi.org/10.1016/j.mce.2015.12.018.

49. Bi W, Gu Z, Zheng Y, Wang L, Guo J, Wu G. Antagonistic and synergistic effects of bone morphogenetic protein $2 / 7$ and all-trans retinoic acid on the osteogenic differentiation of rat bone marrow stromal cells. Develop Growth Differ. 2013;55(9):744-54. https://doi.org/10.1111/dgd.12090.

50. Green A, Rudolph-Stringer V, Straszkowski L, Tjin G, Crimeen-Irwin B, Walia $M$, et al. Retinoic acid receptor $\gamma$ activity in mesenchymal stem cells regulates endochondral bone, angiogenesis, and B lymphopoiesis. J Bone Mineral Res. 2018;33(12):2202-13. https://doi.org/10.1002/jbmr.3558.

51. Balkan W, Rodríguez-Gonzalez M, Pang M, Fernandez I, Troen B. Retinoic acid inhibits NFATc1 expression and osteoclast differentiation. J Bone Miner Metab. 2011;29(6):652-61. https://doi.org/10.1007/s00774-011-0261-0.

52. Cash D, Bock C, Schughart K, Linney E, Underhill T. Retinoic acid receptor alpha function in vertebrate limb skeletogenesis: a modulator of chondrogenesis. J Cell Biol. 1997;136(2):445-57. https://doi.org/10.1083/ jcb.136.2.445.
53. Smit-McBride Z, Modjtahedi S, Cessna C, Telander D, Hjelmeland L, Morse L. In vivo gene expression profiling of retina postintravitreal injections of dexamethasone and triamcinolone at clinically relevant time points for patient care. Invest Ophthalmol Vis Sci. 2011;52(12):8965-78. https://doi. org/10.1167/iovs.10-7084.

54. Hummel R, Ulbrich S, Appel D, Li S, Hirnet T, Zander S, et al. Administration of all-trans retinoic acid after experimental traumatic brain injury is brain protective. Br J Pharmacol. 2020;177(22):5208-23. https://doi.org/10.1111/ bph.15259.

\section{Publisher's Note}

Springer Nature remains neutral with regard to jurisdictional claims in published maps and institutional affiliations.
Ready to submit your research? Choose BMC and benefit from:

- fast, convenient online submission

- thorough peer review by experienced researchers in your field

- rapid publication on acceptance

- support for research data, including large and complex data types

- gold Open Access which fosters wider collaboration and increased citations

- maximum visibility for your research: over $100 \mathrm{M}$ website views per year

At BMC, research is always in progress.

Learn more biomedcentral.com/submissions 